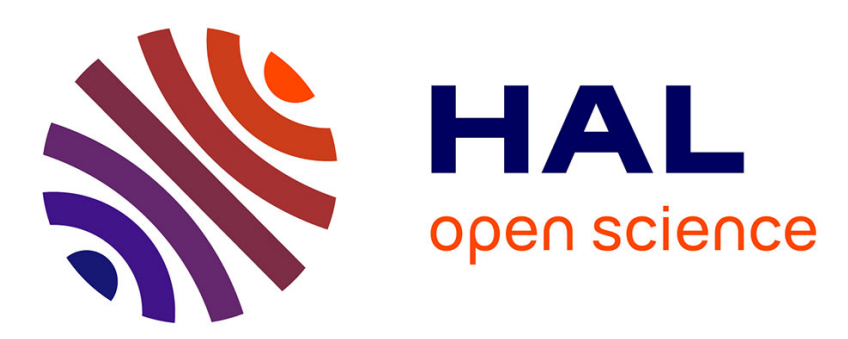

\title{
The Sliding Singular Spectrum Analysis: a Data-Driven Non-Stationary Signal Decomposition Tool
}

\author{
Jinane Harmouche, Dominique Fourer, François Auger, Pierre Borgnat, \\ Patrick Flandrin
}

\section{- To cite this version:}

Jinane Harmouche, Dominique Fourer, François Auger, Pierre Borgnat, Patrick Flandrin. The Sliding Singular Spectrum Analysis: a Data-Driven Non-Stationary Signal Decomposition Tool. IEEE Transactions on Signal Processing, 2017, 10.1109/TSP.2017.2752720 . hal-01589464

\section{HAL Id: hal-01589464 \\ https://hal.science/hal-01589464}

Submitted on 18 Sep 2017

HAL is a multi-disciplinary open access archive for the deposit and dissemination of scientific research documents, whether they are published or not. The documents may come from teaching and research institutions in France or abroad, or from public or private research centers.
L'archive ouverte pluridisciplinaire HAL, est destinée au dépôt et à la diffusion de documents scientifiques de niveau recherche, publiés ou non, émanant des établissements d'enseignement et de recherche français ou étrangers, des laboratoires publics ou privés. 


\title{
The Sliding Singular Spectrum Analysis: a Data-Driven Non-Stationary Signal Decomposition Tool
}

\author{
Jinane Harmouche, Dominique Fourer, Member, IEEE, François Auger, Senior Member, IEEE, \\ Pierre Borgnat, Member, IEEE, and Patrick Flandrin, Fellow, IEEE.
}

\begin{abstract}
Singular Spectrum Analysis (SSA) is a signal decomposition technique that aims at expanding signals into interpretable and physically meaningful components (e.g. sinusoids, noise, etc.). This article presents new theoretical and practical results about the separability of the SSA and introduces a new method called sliding SSA. First, the SSA is combined with an unsupervised classification algorithm to provide a fully automatic data-driven component extraction method for which we investigate the limitations for components separation in a theoretical study. Second, the detailed automatic SSA method is used to design an approach based on a sliding analysis window which provides better results than the classical SSA method when analyzing non-stationary signals with a time-varying number of components. Finally, the proposed sliding SSA method is compared to the Empirical Mode Decomposition (EMD) and to the synchrosqueezed Short-Time Fourier Transform (STFT), applied on both synthetic and real-world signals.
\end{abstract}

Index Terms-Singular Spectrum Analysis, Empirical Mode Decomposition, Synchrosqueezing, non-stationary signals

\section{INTRODUCTION}

$\mathbf{S}$ INGULAR Spectrum Analysis (SSA) [1], [2] is a dataadaptive and non parametric method for time series decomposition and identification. It was introduced in the context of extracting, from noisy sampled observations, experimental data information about an underlying dynamical system [1], [3]. SSA recently received a lot of attention for its ability to provide meaningful results in many application fields, without making any assumption on the processed data. Thus, the main motivation to use SSA is that no stationarity, linearity or normality assumption about the data must be satisfied to provide efficient results. For example, SSA was successfully applied to extract trends from acoustic signals [4] and to expand biological signals into a sum of interpretable components [5][7]. Furthermore, SSA has shown its efficiency for processing stationary and non-stationary signals (using the wide sense stationarity definition [8]) without restriction on the length of the analyzed signal. Simultaneous extraction of trends and amplitude-modulated sinusoids, detection, modeling and structural changes forecasting, are also among the basic capabilities of SSA [9]-[11]. From a SSA perspective, a time series is considered as a sum of deterministic components possibly merged

Manuscript received March 30, 2017; revised August 3, 2017; accepted September 5, 2017. The associate editor coordinating the review of this manuscript and approving it for publication was Prof. Adel Belouchrani.

D. Fourer is with IRCAM, Paris, FR. F. Auger is with IREENA, SaintNazaire, FR. J. Harmouche, P. Borgnat and P. Flandrin are with ENS de Lyon, Laboratoire de Physique, Lyon, FR. with structureless noise. The relevance and the physical meaningfullness of the extracted components is commonly related to the concept of separability [9]. This aims at determining how the components provided by SSA are intercorrelated and can be independently interpreted. Theoretically, one can distinguish weak and strong separability [12], whereas from a practical point-of-view, it is more reasonable to make use of an approximate or asymptotic separability [9]. Several criteria have been proposed to determine the separation/decomposition quality [9], [13], [14]. They are often dedicated to assess the correlation/orthogonality between components, in either time or frequency domain. The result depends on the choice of the SSA parameter called the embedding dimension. For instance, a well-known result is that two sinusoids with very different frequencies and different amplitudes are strongly separable [9]. Sinusoids with close frequencies and different amplitudes are asymptotically separable [9]. This means that they are strongly separable when the embedding dimension and the time series length go to infinity. However, one could be interested in knowing how much two frequencies should be distant for a given value of the embedding dimension. This paper makes a contribution towards answering to this question. In many applications, the components extracted by SSA can be identified as trends, periodic (possibly amplitude-modulated) components or noise. Thus, it shares the same goal as some recently proposed approaches referred to as Empirical Mode Decomposition (EMD) [15], [16]. Intuitively, the question of the limit distance above which two close frequencies are separable applies to the case when the components are modulated. In this article, several separation cases are investigated to concretely justify the SSA behavior when processing some particular non-stationary signals. Furthermore, the question of the robustness of the SSA method to outliers and strong noise, which is full of interest for real-world signals, is also addressed and presented as a particular case study, involving the separation of a periodic component with an impulse signal.

Despite efforts to make spectral methods more robust to outliers [17], [18], in the case of strongly modulated nonstationarity signals, the classical SSA method fails to correctly reconstruct the components. A grouping approach based on the analysis of the singular spectrum is no longer valid because each singular vector can only capture a part of each component. To overcome this limitation, a new algorithm called sliding SSA is introduced and proposes a frame-based SSA (as for the Short-Time Fourier Transform (STFT)), for 
which extracted deterministic components are tracked between adjacent frames. This new method is compared to the classical SSA and provides significantly better results for both stationary and non-stationary signals. The present paper is organized as follows. The next section briefly recalls the basic SSA algorithm and proposes an unsupervised component grouping method based on hierarchical clustering. Section III studies the separability capabilities of the SSA by considering several theoretical and practical cases that are validated through simulations. Section IV introduces a new algorithm called sliding SSA that oversteps the limitations of the classical SSA algorithm when it processes non-stationary multi-components signals. Section $\mathrm{V}$ makes a contribution towards a performance comparison between four mode decomposition techniques: SSA, EMD, synchrosqueezing and the new proposed sliding SSA, which are applied on both synthetic and real-world signals. Finally, this article is concluded in Section VI by future research perspectives.

\section{The Basic Singular Spectrum Analysis method}

\section{A. Algorithm principle}

SSA considers a finite length record of a time series (e.g. a sampled signal) $s=\left\{s_{n}, n=1 \ldots N\right\}$ and aims at decomposing $s$ as a sum of physically interpretable components. This task is generally completed through two successive steps.

\section{1) Decomposition:}

a) Embedding: This step consists in mapping the onedimensional $N$-samples time series into a sequence of $K=$ $N-L+1$ lagged column vectors of length $L$, where $L$ is called the embedding dimension. As a result, we obtain a trajectory matrix expressed as:

$$
\boldsymbol{X}=\left(\begin{array}{cccc}
s_{1} & s_{2} & \ldots & s_{K} \\
s_{2} & s_{3} & \ldots & s_{K+1} \\
\vdots & \vdots & \vdots & \vdots \\
s_{L} & s_{L+1} & \ldots & s_{N}
\end{array}\right)
$$

where each column is obtained by applying a sliding window of length $L$ to the time series. Thus, the trajectory matrix $\boldsymbol{X}$ is a Hankel matrix, meaning that it has equal elements on the diagonals (i.e. $i+j$ is constant, when $i$ and $j$ are row and column indices). The only parameter in this step is the window length $L$, an integer ranging in the interval [2, N-1]. $L$ should be carefully selected because it directly affects the decomposition. The optimal choice depends on the particularity of the time series and on the problem statement [19]. This means that information about the time series structure may help to find an appropriate choice of $L$. However, in most situations this information is not available.

b) Singular value decomposition: The Singular Value Decomposition (SVD) [20] of $\boldsymbol{X}$ (being a real $L \times K$ matrix with rank $R \leq \min (L, K)$ ), expands this matrix into a sum of weighted orthogonal matrices that are not necessarily Hankel, expressed as [21]:

$$
\boldsymbol{X}=\boldsymbol{U} \boldsymbol{\Sigma} \boldsymbol{V}^{T}=\sum_{i=1}^{R} \boldsymbol{X}_{i} \quad \text { with } \boldsymbol{X}_{i}=\sigma_{i} \boldsymbol{u}_{i} \boldsymbol{v}_{\boldsymbol{i}}{ }^{T}
$$

where $\boldsymbol{\Sigma}=\operatorname{diag}\left(\sigma_{1}, \cdots, \sigma_{R}\right), \sigma_{i}$ are the singular values sorted in the descending order, $u_{i}$ and $v_{i}$ are respectively the associated left and right singular vectors corresponding to the columns of the orthogonal matrices $\boldsymbol{U}$ and $\boldsymbol{V}$. The SVD expansion of $\boldsymbol{X}$ can be obtained through the eigendecomposition of the lag-covariance matrix $\boldsymbol{C}=\boldsymbol{X} \boldsymbol{X}^{T}$. This matrix can be factorized as $\boldsymbol{C}=\boldsymbol{U} \boldsymbol{\Lambda} \boldsymbol{U}^{\boldsymbol{T}}$ where $\boldsymbol{\Lambda}$ is the diagonal matrix of eigenvalues. The $i$-th eigenvalue is equal to $\sigma_{i}^{2}$. The right singular vectors $\boldsymbol{V}=\left(\boldsymbol{v}_{\mathbf{1}}, \ldots, \boldsymbol{v}_{\boldsymbol{R}}\right)$ can be deduced from $\boldsymbol{X}$ and $\boldsymbol{U}$ as $\boldsymbol{v}_{\boldsymbol{i}}=\boldsymbol{X}^{\boldsymbol{T}} \boldsymbol{u}_{\boldsymbol{i}} / \sigma_{i}$.

The energy contribution of the $i$-th eigentriple ${ }^{1}$ to the trajectory matrix, given by the ratio $\sigma_{i}^{2} / \sum_{j=1}^{R} \sigma_{j}^{2}$, is called the singular spectrum of the time series.

2) Reconstruction:

a) Grouping: This step consists in splitting the set of elementary matrices $\boldsymbol{X}_{i}(i=1, \ldots, R)$ into $r$ disjoint groups and summing the matrices within each group. The result of this process is the expansion of the trajectory matrix $\boldsymbol{X}$ as $\boldsymbol{X}=\sum_{k=1}^{r} \boldsymbol{X}_{I_{k}}$, where $\boldsymbol{X}_{I_{k}}=\sum_{i \in I_{k}} \boldsymbol{X}_{i}$ is the resulting matrix of group $I_{k}(k=1, \ldots, r)$. The groups $I_{k}$ can be formed based on the information contained into the singular vectors and into the singular spectrum [12], [22]. The singular vectors have a temporal structure and can be considered as time series. For both stationary or non-stationary components, the singular vectors of the same group are assumed to share some features with a signal component. Thus, the grouping is usually made by checking for their correlation degree and by a coherence inspection of the singular spectrum [23]. However, this approach is less valid in the case of strongly non-stationarity signals, because each singular vector is then likely to capture a unique part of the behavior of a component, and the singular vectors within the same group are intended to represent together the whole behavior of one component.

b) Diagonal averaging: If the signal components are separable, the resulting matrices after the grouping step are ideally Hankel [12], [14]. Thus, they correspond to the trajectory matrices of some time series. For real-world signals, this seldom happens, thus the resulting matrices $\boldsymbol{X}_{I_{k}}$ are almost Hankel and the components are approximately separable. The averaging along cross-diagonals of the matrix $\boldsymbol{X}_{I_{k}}$ aims at solving the problem of finding the time series $x^{(k)}$ for which the trajectory matrix of dimension $(L \times K)$ is the closest to $\boldsymbol{X}_{I_{k}}$, in the least-squares sense. In other words, the diagonal averaging of $\boldsymbol{X}_{I_{k}}=\left(x_{i, j}\right)$ provides the elements of the time series $\left\{x_{n}^{(k)}, n=1 \ldots N\right\}$ as:

$$
x_{n}^{(k)}= \begin{cases}\frac{1}{n} \sum_{m=1}^{n} x_{m, n-m+1} & \text { for } 1 \leq n<L \\ \frac{1}{L} \sum_{m=1}^{L} x_{m, n-m+1} & \text { for } L \leq n \leq K \\ \frac{1}{N-n+1} \sum_{m=n-K+1}^{L} x_{m, n-m+1} & \text { for } K+1 \leq n \leq N .\end{cases}
$$

This cross-diagonal averaging (also called Hankelization process) can also be applied to each $\boldsymbol{X}_{i}$ matrix. The resulting time series will hereafter be referred to as elementary components.

This process finally provides an exact expansion of the time series $s$ into $r$ components that satisfies $s_{n}=\sum_{k=1}^{r} x_{n}^{(k)}$.

\footnotetext{
${ }^{1}$ The $i$-th eigentriple is defined by the collection $\left(\sigma_{i}, \boldsymbol{u}_{\boldsymbol{i}}, \boldsymbol{v}_{\boldsymbol{i}}\right)$.
} 


\section{B. Automatic grouping of components}

Since numerous specific component grouping methods were separately proposed for SSA to reconstruct sinusoids [23], [24], trends [25] or noise [6], [9], all of them suffer of a lack of flexibility and require to be manually adapted to the analyzed signal to provide meaningful results. More recently, efforts aim at making the SSA fully automatic [26]-[28] and adaptive for any component type.

Thus, we propose to use agglomerative hierarchical clustering [29] to extract the physically interpretable components of a time series, regardless of the component types [30]. This approach pioneered in [31]-[33], contrarily to classical methods, first reconstructs each elementary component by the Hankelization process and then apply the grouping. The algorithm is initialized by assigning each reconstructed elementary component (associated to a eigentriple) to a distinct class [29]. Then, the algorithm looks for the two nearest classes and merges them to build a new class. This operation is iterated until the desired number of classes or the maximum dissimilarity between two classes is reached. To compare two classes $C_{i}$ and $C_{j}$ containing several time series, the dissimilarity is defined as the minimal distance between two distinct time series of each class

$$
D\left(C_{i}, C_{j}\right)=\min _{x \in C_{i}, y \in C_{j}} d(x, y)
$$

where the chosen distance function $d: \mathbb{R}^{N} \times \mathbb{R}^{N} \rightarrow[0,1]$ is defined as

$$
\begin{gathered}
d(x, y)=1-\frac{|<x, y\rangle \mid}{\|x\|\|y\|}, \\
\text { with }<x, y>=x^{T} y \text { and }\|x\|=\sqrt{<x, x>}
\end{gathered}
$$

but can also be replaced by the weighted correlation (wcorrelation) as defined in [33].

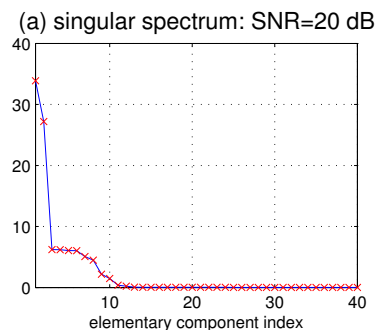

(c) component $1: \mathrm{QRF}=26 \mathrm{~dB}$

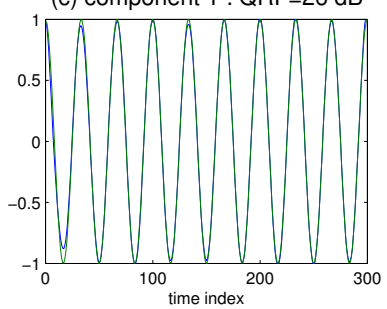

(b) dendrogram

(d) component $2: \mathrm{QRF}=19 \mathrm{~dB}$

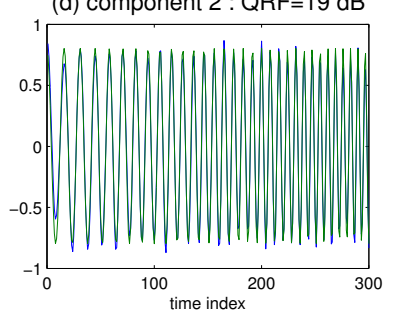

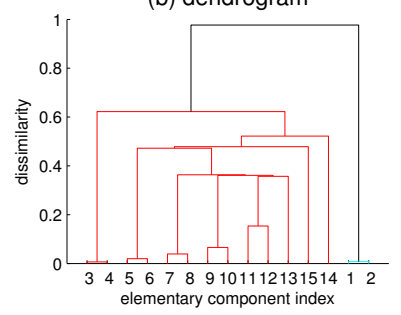

Fig. 1. Decomposition of a signal of $N=300$ samples made of a sinusoid with normalized frequency $\lambda_{0}=0.03$ and a linearly frequency-modulated sinusoid with amplitude $H=0.8$ and frequency varying from 0.06 to 0.15 merged with a white Gaussian noise with a SNR equal to $20 \mathrm{~dB}$. This result is obtained with the proposed algorithm with $L=40$ and for a maximum number of classes set to $r=2$ (a distinct color per class).
This algorithm provides a binary tree that can be called a dendrogram, which shows at each iteration the result of the merging process. An example is presented in Fig. 1 (b) where the $\mathrm{X}$-axis shows the components indices and the Y-axis shows the maximal distance between two elementary components of the same class. The reconstructed components associated to each class are displayed in Figs. 1 (c) and (d). The Quality of Reconstruction Factor (QRF) of an estimated component $\hat{x}$ relative to reference $x$ is given by

$$
\operatorname{QRF}(\hat{x}, x)=20 \log _{10}\left(\frac{\|x\|}{\|x-\hat{x}\|}\right) \text {. }
$$

As shown in Fig. 1, the proposed algorithm allows to recover the elementary components despite the presence of noise (component QRF is higher or equal to the input SNR).

Our numerical experiments show that hierarchical clustering often obtains better results than with the k-means algorithm [34] as proposed in [31]. Furthermore, the results provided by the correlation function defined by Eq. (5) obtains as good results as when using the w-correlation in practice. Thus, the entire proposed automatic SSA method which combines basic SSA and automatic grouping can be formalized by Algorithm 1 for which a MATLAB implementation is proposed in [35].

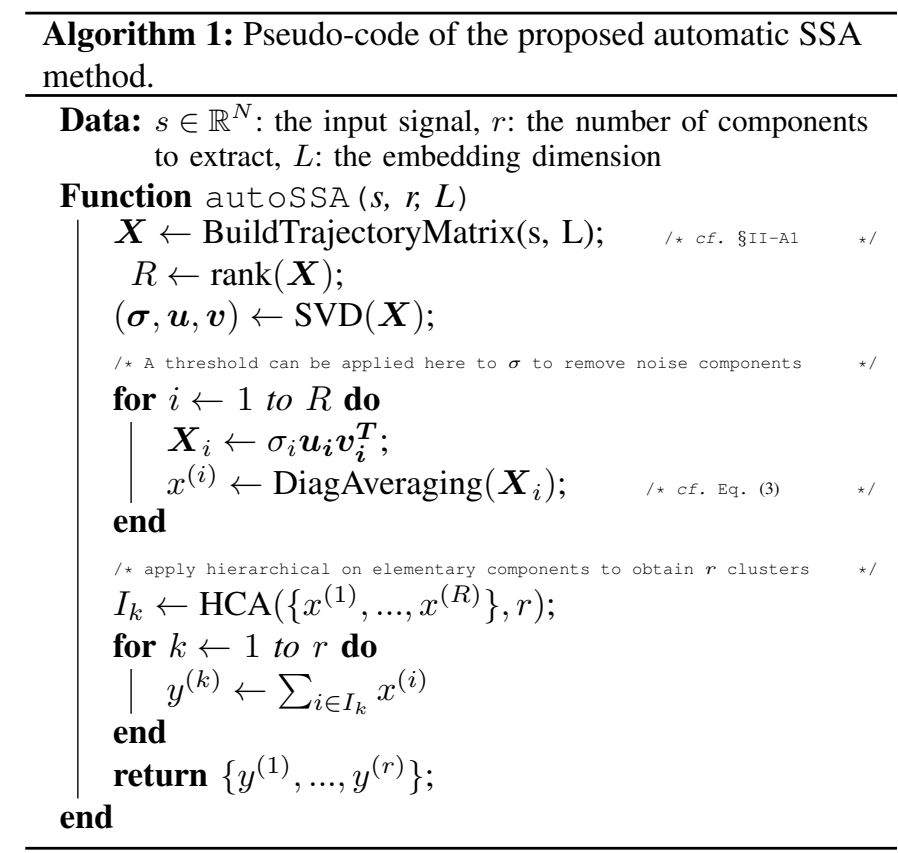

\section{THEORETICAL AND PRACTICAL RESULTS FOR COMPONENTS SEPARATION}

Several separability degrees from both theoretical and practical viewpoints can be defined [12]:

- weak separability: two distinct time series $x^{(i)}$ and $x^{(j)}$, with trajectory matrices $\boldsymbol{X}_{i}$ and $\boldsymbol{X}_{j}$, are said to be weakly $L$-separable if all pairwise inner product of the rows and columns of $\boldsymbol{X}_{i}$ and $\boldsymbol{X}_{j}$ are equal to zero. This means that any subseries of length $L$ of $x^{(i)}$ is orthogonal by any subseries of the same length of $x^{(j)}$.

- strong separability: $x^{(i)}$ and $x^{(j)}$ are said to be strongly $L$-separable if, in addition to the weak separability condition, the SVD of $X_{i}$ and $X_{j}$ have distinct singular values. 


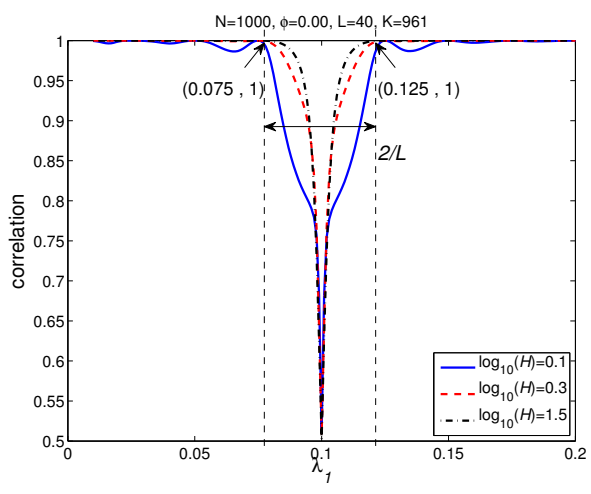

(a) Frequency separability for different $H$ values.

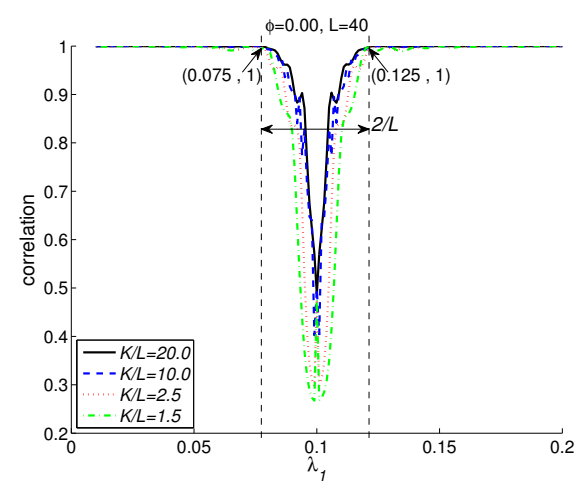

(b) Frequency separability for different $K$ values.

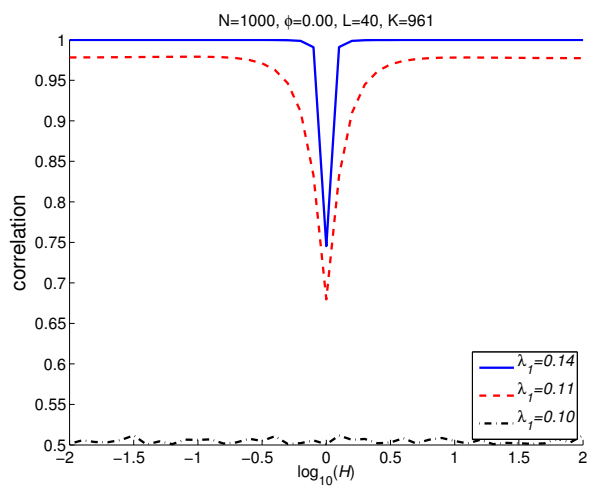

(c) Amplitude separability for different $\lambda_{1}$ values.

Fig. 2. Average correlation between the sinusoids in signal model of Eq. (9) for close frequencies. (a) details the correlation with $\log _{10}(H) \in\{0.1,0.3,1.5\}$ and (b) with $L=40$ and $N$ varying such as $K \in\{800,400,200,100\}$. (c) details the correlation for close amplitudes with $\lambda_{1} \in\{0.10,0.11,0.14\}$.

As the strong separability is never verified for real-world series, two practical degrees of separability are defined as follows [12]:

- approximate separability: two time series $x^{(i)}$ and $x^{(j)}$ are said to be approximately $L$-separable if all pairwise inner products of the rows and columns of $\boldsymbol{X}_{i}$ and $\boldsymbol{X}_{j}$ are close to zero.

- asymptotic separability: two components $x^{(i)}$ and $x^{(j)}$ are said to be asymptotically $L$-separable if they are approximately $L$-separable when $N \rightarrow \infty$ and $L \rightarrow \infty$.

\section{A. Separation of two sinusoids}

Two sinusoids with different amplitudes and different (even very close) frequencies are known to be asymptotically separable [12]. The question here is: for given $L$ and $N$, what is the minimum gap required between two frequencies in order to separate them? It has been shown theoretically and validated experimentally [36] that each singular value of the trajectory matrix can be approximated by averaging a suitable portion of the periodogram of the analyzed time series. More precisely, let $s=\left\{s_{n}, n=1 \ldots N\right\}$ be a stationary zero mean, time series and its $L \times K$ trajectory Hankel matrix $\boldsymbol{X}$ defined as in Eq. (1). We define the vectors $\vec{s}=$ $\left(s_{1}, s_{2}, \ldots, s_{K}\right)^{T}$ and $\overrightarrow{\hat{s}}=\left(\hat{s}_{1}, \hat{s}_{2}, \ldots, \hat{s}_{K}\right)^{T}$ as being a subseries of $s$ of length $K$ and its unitary Fourier transform can be computed as $\hat{s}_{k}=\frac{1}{\sqrt{K}} \sum_{n=1}^{K} s_{n} \mathbf{e}^{-2 j \pi \frac{(n-1) k}{K}}$. Thus, the periodogram of $s$ is defined as $\left(\left|\hat{s}_{0}\right|^{2},\left|\hat{s}_{1}\right|^{2}, \ldots,\left|\hat{s}_{K-1}\right|^{2}\right)$ and verifies $\left|\hat{s}_{k}\right|^{2}=\left|\hat{s}_{K-k+1}\right|^{2}$, if $\vec{s} \in \mathbb{R}^{K}$. Under the condition $L \ll K$, each eigenvalue $\sigma_{i}^{2}$ of the normalized covariance matrix $\boldsymbol{C}=\frac{1}{K} \boldsymbol{X} \boldsymbol{X}^{T}$ can be approximated by the mean value of a portion of the power spectrum $\overrightarrow{\hat{s}}$, whose length can roughly be defined as $l=\left\lfloor\frac{K}{L}\right\rfloor,(\lfloor x\rfloor$ being the integer part of a real number $x$ ) [36]:

$$
\bar{\sigma}_{i}^{2}=\frac{1}{l} \sum_{j=(i-1) l}^{i l-1}\left|\hat{s}_{j}\right|^{2}, \quad i=1, \ldots, L .
$$

Now, one can deduce that each eigentriple requires about $K / L$ values of $\hat{s}$ which has a resolution of $1 / K$ cycles per sample. Hence, one can conclude that the minimal distance between two frequencies is $\Delta \lambda_{\min }=\frac{K}{L} \times \frac{1}{K}=1 / L$. This statement can be validated by numerical simulations using the following signal model

$$
s_{n}=\underbrace{\cos \left(2 \pi \lambda_{0} n\right)}_{x_{n}^{(1)}}+\underbrace{H \cos \left(2 \pi \lambda_{1} n+\phi\right)}_{x_{n}^{(2)}}, \quad n=1 \cdots N .
$$

SSA is applied to this time series, while $\lambda_{0}=0.1, \lambda_{1}$ belongs to $[0.01,0.2]$ with $L=40$. For this experiment, we use the proposed automatic grouping approach. The reconstruction quality, presented in Fig. 2, is computed by the average correlation between the resulting components and the original ones as $\frac{1}{2}\left(\frac{\left|<x^{(1)}, y^{(1)}>\right|}{\left\|x^{(1)}\right\| \mid\left\|y^{(1)}\right\|}+\frac{\left|<x^{(2)}, y^{(2)}>\right|}{\left\|x^{(2)}||\right\| y^{(2)} \|}\right)$ where $y^{(1)}$ (resp. $\left.y^{(2)}\right)$ is the reconstructed component associated to $x^{(1)}$ (resp. $\left.x^{(2)}\right)$. Results show that SSA manages to separate the two sinusoids except in two cases:

$1)$ they have equal amplitudes $\left(\log _{10}(H) \approx 0\right)$. The singular values of both signals are then almost identical and cannot be distinguished in the singular spectrum,

$2)$ their frequencies are too close $\left(\lambda_{1} \approx 0.1\right)$ then the minimal distance condition for separability is not verified.

\section{B. Separation of a sinusoid and a chirp}

In order to extend the analysis to non-stationary signals, let's consider the following signal model

$$
\begin{aligned}
s_{n} & =\cos \left(2 \pi \lambda_{0} n\right)+H \cos (\varphi(n)), n=1, \ldots, N(10) \\
\text { with } \varphi(n) & =2 \pi\left(\lambda_{1} n+(\delta \lambda / 2 N) n^{2}\right)
\end{aligned}
$$

where $H \in[0.01,100]$ and $\delta \lambda$ varies in the interval $[0,0.2]$. According to whether a spectral overlapping between the sinusoid and the chirp exists or not, three situations are possible: (1) $\lambda_{0}<\lambda_{1}$, (2) $\lambda_{1} \leq \lambda_{0} \leq \lambda_{1}+\delta \lambda$ and (3) $\lambda_{1}+\delta \lambda<\lambda_{0}$. In order to characterize the separability, $\lambda_{1}$ is set to a specific value in each of the three cases so that the reconstruction can be evaluated with respect to $\delta \lambda$ and $H$ as displayed in Fig. 3 , obtained with $L=40$ and $N=500$. The separability is measured as in the stationary case ( $c f$. Section III-A), using the average correlation between the two original components and the reconstructed ones. For components reconstruction, the proposed automatic method ( $c f$. Section II-B) is compared with a supervised approach ( $c f$. Fig.3) which consists in grouping 

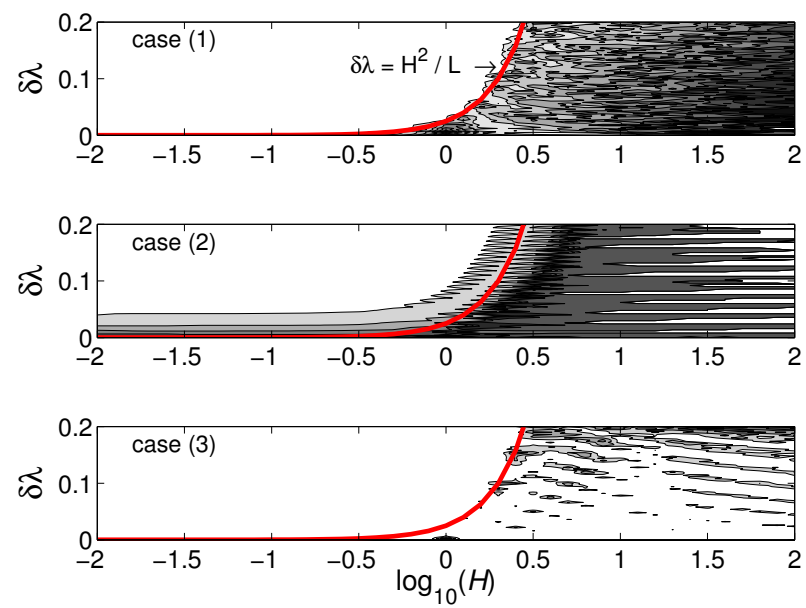

(a) supervised grouping
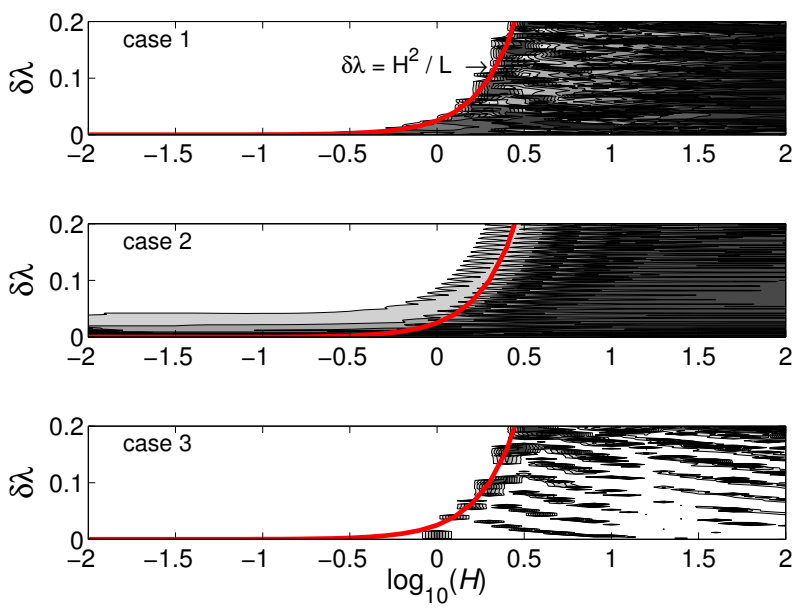

(b) automatic (unsupervised) grouping

Fig. 3. Average correlation between the signal components and their reconstructions using a supervised grouping method (a) and the proposed automatic grouping method (b). In case $1, \lambda_{0}=0.1$ and $\lambda_{1}=0.11$, case $2, \lambda_{1}=0.11$ and $\lambda_{0}=\lambda_{1}+\delta \lambda / 2$, case $3, \lambda_{0}=0.31$ and $\lambda_{1}=0.1$.

the elementary components which have the minimal square relative error with the reference components (assumed known).

Hence, the relative error between an elementary component $y$ and a reference component $x$ is computed as $\sum_{n} \mid x_{n}-$ $\left.y_{n}\right|^{2} / \sum_{n} x_{n}^{2}$. In the three cases, we observe a transition from an area where the reconstruction succeeds everywhere (white area where correlation is close to one) to an area where it fails (case 1) or can fail (cases 2 and 3). The red curve shows the border between the two areas. Note that in the situation where there is a spectral overlap between the signal components (case 2), the shaded zone is clearly enlarged exceeding the plotted curve. The shown border corresponds to the situation where the singular values associated to the chirp reach in magnitude those associated to the sinusoid. Indeed, it follows from the spectral interpretation given by Eq. (8), that a real chirp having a flat spectrum with bandwidth $\delta \lambda$ evenly spreads its spectrum across $\lfloor L \delta \lambda\rfloor$ eigenvalues. So, the chirp is associated to a set of eigenvalues, whose level is approximately $\left(H^{2} / 2\right) /(L \delta \lambda)$ [30]. Similarly, the amplitude of the pair of eigenvalues associated to the sinusoid is $1 / 2$. The equality between both quantities leads to $\delta \lambda=H^{2} / L$, which corresponds to the red curve shown in Fig. 3.

This figure shows that the proposed automatic grouping achieves optimal performance, comparable to the supervised approach, while respecting the theoretical boundary. A major difference however exists with the supervised approach in the case of spectral overlap (case 2): the ambiguity area is transformed into an area where the separation fails everywhere.

\section{Separation of a sinusoid and an impulse}

SSA is commonly defined for real-valued time series. This does not preclude the application of SSA to a complex-valued trajectory matrix [37]. The complex SSA algorithm is the same as the basic SSA, with real-valued singular values, complexvalued eigenvectors and where the transpose operator turns into the Hermitian transpose operator.

The effect of an impulse on the reconstruction of a periodic component will be studied in this section. Let's consider the complex-valued signal model defined as

$$
s_{n}=\mathbf{e}^{j(n \theta+\phi)}+H \delta\left(n-n_{0}\right), \quad n=1, \ldots, N
$$

where $j^{2}=-1, \theta=2 \pi \lambda$ is the normalized angular frequency and $H \delta(n)$ is the Dirac delta function with amplitude $H$. We assume that $n_{0}>L$, where $n_{0}$ is the time localization of the impulse appearance. Let $\boldsymbol{X}$ be the trajectory matrix of the series $x_{n}=\mathbf{e}^{j(n \theta+\phi)}$,

$$
\boldsymbol{X}=\mathbf{e}^{j \phi}\left(\begin{array}{cccc}
\mathbf{e}^{j \theta} & \mathbf{e}^{2 j \theta} & \ldots & \mathbf{e}^{K j \theta} \\
\mathbf{e}^{2 j \theta} & \mathbf{e}^{3 j \theta} & \ldots & \mathbf{e}^{(K+1) j \theta} \\
\vdots & \vdots & \vdots & \vdots \\
\mathbf{e}^{L j \theta} & \mathbf{e}^{(L+1) j \theta} & \ldots & \mathbf{e}^{N j \theta}
\end{array}\right)
$$

and let $\boldsymbol{Y}$ be the trajectory matrix of $y_{n}=H \delta\left(n-n_{0}\right)$ when $n_{0}>L$ and $L<K$

$$
\begin{gathered}
\boldsymbol{Y}=H \cdot \\
\left(\begin{array}{cccccccccccc}
0 & \ldots & \ldots & 0 & 0 & \ldots & \ldots & 1 & 0 & \ldots & \ldots & 0 \\
\vdots & \ddots & & \vdots & \vdots & & . & \vdots & \vdots & \ddots & & \vdots \\
\vdots & & \ddots & \vdots & \vdots & . & & \vdots & \vdots & & \ddots & \vdots \\
0 & \ldots & \ldots & 0 & 1 & \ldots & \ldots & 0 & 0 & \ldots & \ldots & 0
\end{array}\right) .
\end{gathered}
$$

Now, it can be shown that $\operatorname{rank}(\boldsymbol{X})=1$ (since each line vector of $\boldsymbol{X}$ is equal to the line above multiplied by $\left.\mathbf{e}^{j \theta}\right)$ and $\operatorname{rank}(\boldsymbol{Y})=L$. Then, we can define $\boldsymbol{u}_{\mathbf{1}}=$ $\left[\begin{array}{lllll}1 & \mathbf{e}^{j \theta} & \mathbf{e}^{2 j \theta} & \ldots & \mathbf{e}^{j(L-1) \theta}\end{array}\right]^{T}$ as a non-unitary singular vector spanning the column space of $\boldsymbol{X}$ associated to the singular value $\sigma_{1}=\mathbf{e}^{j(\theta+\phi)}$ such that $\boldsymbol{X}=\sigma_{1} \boldsymbol{u}_{\mathbf{1}} \boldsymbol{v}_{\mathbf{1}}^{\dagger}$, with $\boldsymbol{v}_{\mathbf{1}}=\left[\begin{array}{lllll}1 & \mathbf{e}^{-j \theta} & \mathbf{e}^{-2 j \theta} & \ldots & \mathbf{e}^{-j(K-1) \theta}\end{array}\right]^{T} \quad\left(\boldsymbol{X}^{\dagger}\right.$ being the transpose conjugate of $\boldsymbol{X})$. We also have, $\boldsymbol{Y} \boldsymbol{Y}^{\dagger}=H^{2} \boldsymbol{I}_{L}$ where $\boldsymbol{I}_{L}$ is the $(L \times L)$ identity matrix. Hence, one can write

$$
\begin{aligned}
(\boldsymbol{X}+\boldsymbol{Y})(\boldsymbol{X}+\boldsymbol{Y})^{\dagger}= & \boldsymbol{X} \boldsymbol{X}^{\dagger}+\boldsymbol{X} \boldsymbol{Y}^{\dagger}+\boldsymbol{Y} \boldsymbol{X}^{\dagger}+\boldsymbol{Y} \boldsymbol{Y}^{\dagger} \\
= & K \boldsymbol{u}_{\mathbf{1}} \boldsymbol{u}_{\mathbf{1}}^{\dagger}+H \mathbf{e}^{j\left(n_{0} \theta+\phi\right)} \boldsymbol{u}_{\mathbf{1}} \boldsymbol{u}_{\mathbf{1}}^{\dagger} \\
& +H \mathbf{e}^{-j\left(n_{0} \theta+\phi\right)} \boldsymbol{u}_{\mathbf{1}} \boldsymbol{u}_{\mathbf{1}}^{\dagger}+H^{2} \boldsymbol{I}_{L} \\
= & \boldsymbol{U} \boldsymbol{\Sigma} \boldsymbol{U}^{\dagger}
\end{aligned}
$$




$$
\boldsymbol{u}_{\mathbf{1}}\left[\boldsymbol{Y}^{\dagger} \boldsymbol{u}_{\mathbf{1}}\right]^{\dagger}=H\left(\begin{array}{cccccccccccc}
0 & \ldots & 0 & \mathbf{e}^{-j(L-1) \theta} & \mathbf{e}^{-j(L-2) \theta} & \ldots & \mathbf{e}^{-2 j \theta} & \mathbf{e}^{-j \theta} & 1 & 0 & \ldots & 0 \\
0 & \ldots & 0 & \mathbf{e}^{-j(L-2) \theta} & \mathbf{e}^{-j(L-3) \theta} & \ldots & \mathbf{e}^{-j \theta} & 1 & \mathbf{e}^{j \theta} & 0 & \ldots & 0 \\
0 & \ldots & 0 & \mathbf{e}^{-j(L-3) \theta} & \mathbf{e}^{-j(L-4) \theta} & \ldots & 1 & \mathbf{e}^{j \theta} & \mathbf{e}^{2 j \theta} & 0 & \ldots & 0 \\
\vdots & & \vdots & \vdots & \vdots & . & \vdots & \vdots & \vdots & \vdots & & \vdots \\
0 & \ldots & 0 & \mathbf{e}^{-j \theta} & 1 & \ldots & \mathbf{e}^{j(L-4) \theta} & \mathbf{e}^{j(L-3) \theta} & \mathbf{e}^{j(L-2) \theta} & 0 & \ldots & 0 \\
0 & \ldots & 0 & 1 & \mathbf{e}^{j \theta} & \ldots & \mathbf{e}^{j(L-3) \theta} & \mathbf{e}^{j(L-2) \theta} & \mathbf{e}^{j(L-1) \theta} & 0 & \ldots & 0
\end{array}\right) .
$$

where $\boldsymbol{U}=\left(\boldsymbol{u}_{1}, \boldsymbol{u}_{2}, \ldots, \boldsymbol{u}_{\boldsymbol{L}}\right)$ is a non-unitary eigenvectors matrix and $\boldsymbol{\Sigma}=\operatorname{diag}\left(K+2 H \cos \left(n_{0} \theta+\phi\right)+\right.$ $\left.H^{2}, H^{2}, H^{2}, \ldots, H^{2}\right)$. It follows that the impulse does not affect the singular vector $\boldsymbol{u}_{1}$ associated to the sinusoidal component $x_{n}$ but adds to the singular spectrum $(L-1)$ nonzero singular values.

Now, it is interesting to investigate the impulse component effect on the sinusoidal component reconstruction process. This is obtained by averaging along the crossdiagonals (Hankelization process) of the matrix $\boldsymbol{u}_{\mathbf{1}}\left[\boldsymbol{X}^{\dagger} \boldsymbol{u}_{\mathbf{1}}+\right.$ $\left.\boldsymbol{Y}^{\dagger} \boldsymbol{u}_{\boldsymbol{1}}\right]^{\dagger}$. The averaging along the cross-diagonals of $\boldsymbol{u}_{\mathbf{1}}\left[\boldsymbol{X}^{\dagger} \boldsymbol{u}_{\mathbf{1}}\right]^{\dagger}$ gives the sinusoidal part. Now let's calculate the impulse part $\boldsymbol{u}_{\mathbf{1}}\left[\boldsymbol{Y}^{\dagger} \boldsymbol{u}_{\mathbf{1}}\right]^{\dagger}$. We obtain $\boldsymbol{Y}^{\dagger} \boldsymbol{u}_{\mathbf{1}}=$ $\left.\begin{array}{lllllllllll}0 & \ldots & 0 & \mathbf{e}^{j(L-1) \theta} & \mathbf{e}^{j(L-2) \theta} & \ldots & \mathbf{e}^{j \theta} & 1 & 0 & \ldots & 0\end{array}\right]$. The first non-zero element $\mathbf{e}^{j(L-1) \theta}$ is at position $n_{0}-L+1$ and the last one is at position $n_{0}$. Thus $\boldsymbol{u}_{1}\left[\boldsymbol{Y}^{\dagger} \boldsymbol{u}_{1}\right]^{\dagger}$ can be expressed by Eq. (12).

Thus, the cross-diagonals averaging of Eq. (12) provides the following $N$-length column vector

$$
\begin{array}{r}
\boldsymbol{q}=H\left[0 \ldots 0 \alpha_{1} \mathbf{e}^{-j(L-1) \theta} \alpha_{2} \mathbf{e}^{-j(L-2) \theta} \ldots \alpha_{L} \ldots\right. \\
\left.\alpha_{2} \mathbf{e}^{j(L-2) \theta} \alpha_{1} \mathbf{e}^{j(L-1) \theta} 0 \ldots 0\right]^{T}
\end{array}
$$

where the $\alpha_{i}$ are obtained by applying Eq. (3) on the identity matrix $\boldsymbol{I}_{L}$. Assuming that $L<n_{0}$, if $L<\left(n_{0}-L+1\right)$ and $\left(n_{0}+L-1\right)<K$, then $\alpha_{i}=i / L, \forall i \in[1, L]$, since $i$ cross-diagonal non-zero elements are summed.

This vector has non-zero elements at positions that lie in the interval $\left[n_{0}-L+1, n_{0}+L-1\right]$, and the maximal amplitude is reached at $n_{0}$. The real part of $\boldsymbol{q}$ is oscillating at angular frequency $\theta$ and is symmetric with respect to $n_{0}$. As a consequence, the reconstruction through SSA of the real-part of the series $s_{n}$ provides the component

$$
z_{n}=\cos (n \theta+\phi)+\frac{2}{L} \operatorname{Re}\left(\boldsymbol{q}_{n}\right), \quad n=1, \ldots, N,
$$

where $\boldsymbol{q}_{n}$ denotes the $n$-th sample of the time series given by Eq. (17). The ratio $1 / L$ is due to the normalization of the singular vector $\boldsymbol{u}_{1}$. The factor 2 accounts for the pair of singular vectors associated to the sinusoidal component in the time domain. $\operatorname{Re}(z)$ denotes the real part of $z$.

Fig. 4 illustrates this result by displaying the average correlation between the reconstructed components and the reference signals using SSA with the proposed grouping method on a real signal made of a sinusoid and an impulse expressed as $s_{n}=\cos \left(2 \pi \lambda_{0} n\right)+H \delta\left(n-n_{0}\right)$ with $\lambda_{0}=0.025, n_{0} \in[L, K]$, $N=500$ and $L=40$. This result shows that the quality of reconstruction of both components depends on the following inequality

$$
1+\frac{2 H}{K} \cos \left(n_{0} \theta+\phi\right)>0
$$

which can be deduced from the theoretical singular spectrum of a sinusoid merged with an impulse given by Eq. (16). Hence, if $H>K / 2$, then the inequality (19) is not verified for the values of $n_{0}$ where the cosine function takes negative values. The result is a black area which corresponds to an average correlation of the reconstructed components with the reference signals, close to zero.

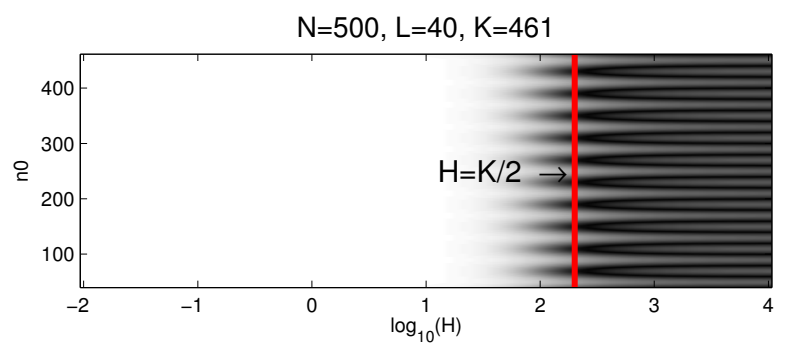

Fig. 4. Average correlation between the signal reconstructed components of a signal made of a sinusoid and of an impulse using the proposed unsupervised SSA method.

\section{THE SLIDING SSA METHOD: AN EMD-LIKE TOOL}

Basically, SSA's theory has a close connection with Fourier analysis and it finds a solid mathematical background on stationary time series analysis. However, this does not exclude the applications of SSA to the analysis of non stationary series since SSA has always been very appealing as an exploratory data-adaptive multicomponent analysis technique. Although methods like EMD and Synchrosqueezing share with SSA the goal of multicomponent signal analysis, SSA has been rarely associated with these methods but rather with subspace methods and multivariate statistics [21]. This is perhaps due to the use of the SVD which is the main element of SSA's algorithm. Now, we illustrate how classical SSA decomposes a multi-components signal and we introduce a new algorithm, called sliding SSA which makes it more adaptive to process non stationary series.

Let consider a $N=1000$ samples multicomponent signal displayed in Fig. 5(a) and expressed as

$$
\begin{aligned}
s_{n} & =x_{n}^{(1)}+x_{n}^{(2)}+x_{n}^{(3)}, \\
\text { where } \quad x_{n}^{(1)} & =a_{1} \sin \left(2 \pi \lambda_{1} n\right) \\
x_{n}^{(2)} & =a_{2} \sin \left(2 \pi \lambda_{2} n+30 \sin \left(2 \pi \lambda_{c} n\right)\right) \\
x_{n}^{(3)} & =a_{3} \sin \left(2 \pi \lambda_{3} n+52.5 \sin \left(2 \pi \lambda_{c} n\right)\right),
\end{aligned}
$$




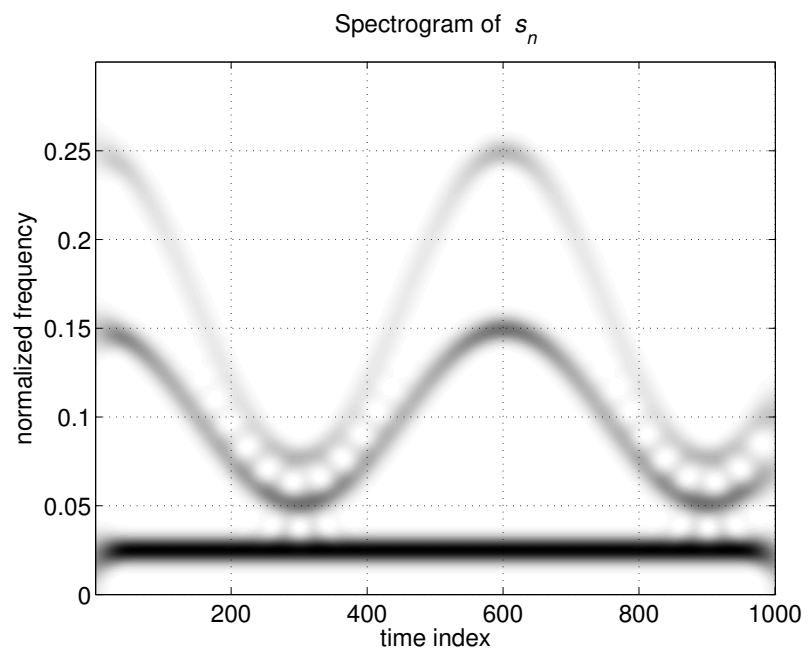

(a) reference signal
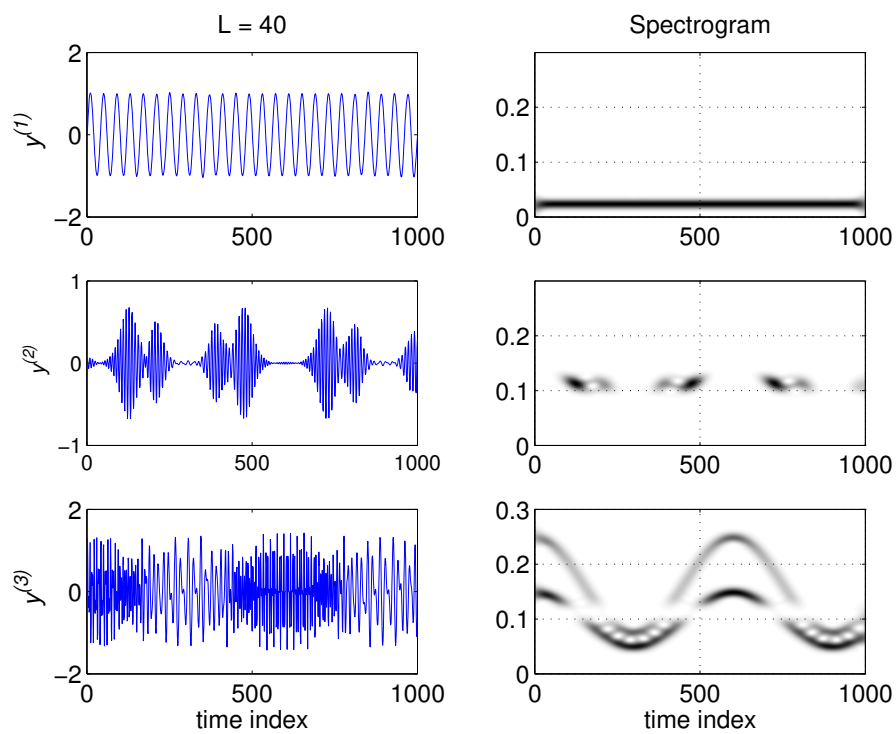

(b) resulting components provided by automatic SSA

Fig. 5. Spectrogram of a multicomponent signal $s_{n}$ made of two sinusoidally frequency modulated chirps and a pure sinusoid (a) and its reconstructed components provided by automatic SSA applied to obtain $r=3$ components denoted $y^{(1)}, y^{(2)}$ and $y^{(3)}$.

with $a_{1}=1, a_{2}=0.8, a_{3}=0.63, \lambda_{1}=0.025, \lambda_{2}=0.01$, $\lambda_{3}=0.1625$ and $\lambda_{c}=\frac{1}{600}$. The proposed automatic SSA method ( $c f$. Algorithm 1) is applied on $s_{n}$ to obtain three components. The resulting components with their respective spectrogram are displayed in Fig. 5, for $L=40$. Our experiments show that the classical SSA fails to recover each elementary component for any value of $L$. It can only recover the pure sinusoid and a relatively narrow-band component, while the two chirps remain merged into the third resulting component.

Obtaining the desired components through an unsupervised grouping of the elementary components is not trivial since each elementary component reflects the signal behavior into a narrow band of nearly $K / L$ cycles per sample [36]. Hence, the correlation between the elementary components of a nonstationary signal component is quite weak. The singular spectrum and its estimation computed using Eq. (8) are shown in Fig. 6. However, it might be possible to make the proper grouping by visual identification and manual assignment of the different components. This task fails if an elementary component is a mixing of contributions from different signal components, like the situation illustrated in Fig. 5.

Let's get back to the question in the header of this section. A mode is formally intended to mean an amplitude-frequency modulated (AM-FM) wave. Comparing SSA to its analogues, among which the EMD and the synchrosqueezing, SSA requires an extensive supervised grouping work to be considered as a mode decomposition-like tool. The proposed approach to bypass this difficulty is: instead of applying SSA directly to the whole signal, we rather propose to consider a sliding window into which automatic SSA is be applied. This algorithm involves linkage between the subcomponents of successive frames (i.e. time series fragments), which is performed by tracking a similarity measure between the subcomponents. The next section details the proposed sliding SSA.

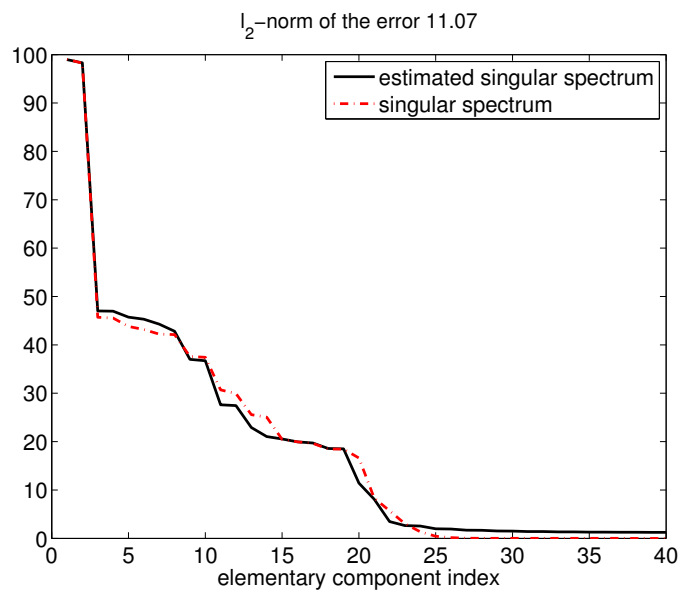

Fig. 6. Singular spectrum $(L=40)$ and its image estimated through the signal energy spectral density.

\section{A. The sliding SSA algorithm}

Sliding SSA contributes to extend the SSA separation capabilities to non-stationary signals with AM-FM components. This approach is motivated by the following issues:

- an unsupervised grouping approach cannot be used due to the low level of the interdependence between the elementary components belonging to one signal component;

- a mixing mode problem may occur due to spectral overlap between different signal components;

- an appearance or vanishing of a component is possible; a sliding locally-adaptive SSA is required in this case.

Thus, our proposal formulated by Algorithm 2, uses the function autoSSA $(x, r, L)$ to extract $r$ components from a time series $x$, through the automatic SSA method (cf. Algorithm 1). At the first position of the sliding window (i.e. when $p=1$ ), the estimated components denoted $\hat{x}_{n}^{(i)}$ are directly initialized 
with the result provided by autoSSA for the samples ranging in $\left[1, n_{c}\right]$ ( $n_{c}$ being the sample index at the center of the current frame).

Then, for the next positions (i.e. $p>1, p$ being the current position index), the current estimated components $\tilde{x}^{(j)}$ provided by autoSSA ( $c f$. Algorithm 1), are matched with the previously estimated ones $\hat{x}_{p-\Delta+n}^{\left(j^{\prime}\right)}$ (after considering a delay $\Delta$ ). The matching is completed through minimization of a given distance metric function denoted $\operatorname{dist}(\hat{x}, \tilde{x})$ which can be defined as Eq. (5) or as the Euclidean distance. For the matching procedure, a variable $J$ is used to store the previously associated components $j^{\prime}$ to ensure that, each one is only associated once.

If the number of component increases, any $j^{\prime} \in[1, \max (r)]$ is choosen among the non-affected components of $\hat{x}$ and is associated to the current component $x^{(j)}$. If this number is identical or decreases, each current component $x^{\tilde{(j)}}$ is necessary associated to a previously existing one of $\hat{x}^{(j)}$.

When the last position is reached (i.e. $p=N-W+1$ ), all the samples ranging in $\left[n_{c}, W\right]$ of each $\tilde{x}_{n}^{(j)}$ are stored in their corresponding associated component.

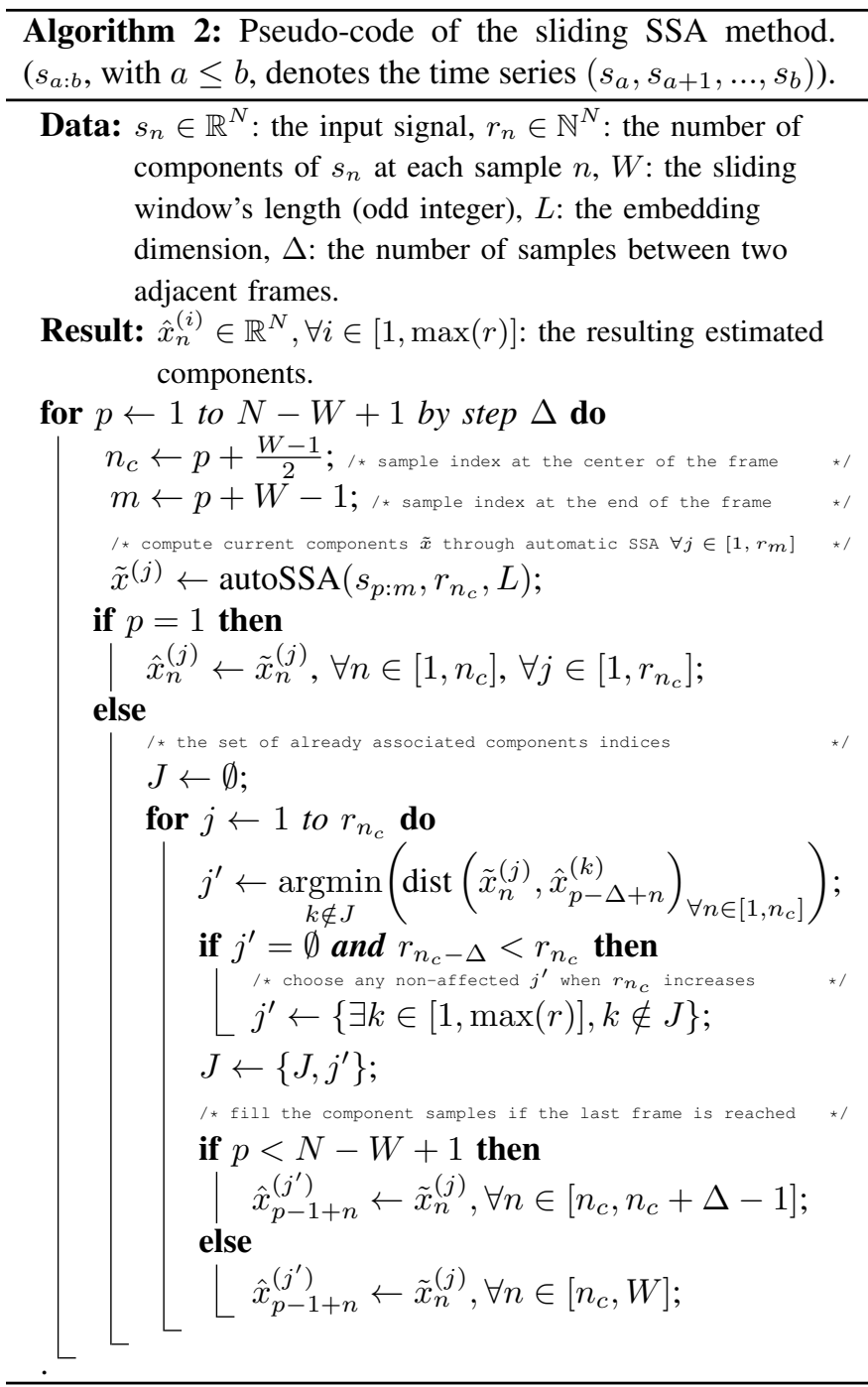

\section{B. Numerical results}

1) Example 1: Consider the signal given by Eq. (20). Its parameters are chosen such that the minimal frequency gap between two components is equal to the critical value $\Delta \lambda_{\min }=$ 0.025 found in Section III-A and the logarithmic amplitude ratio is equal to 0.1 . Fig. 7 shows the signal components and the components reconstructed using sliding SSA with $r_{n}=3$, $\forall n \in[1, N]$, while the embedding dimension $L$ and the sliding window $W$ are set to 40 and 91 respectively. The window moves one sample at each position, $\Delta=1$. The linkage step is based on the Euclidean distance between the samples of the preceding and the current subcomponents located at indices in $[46,76]$.
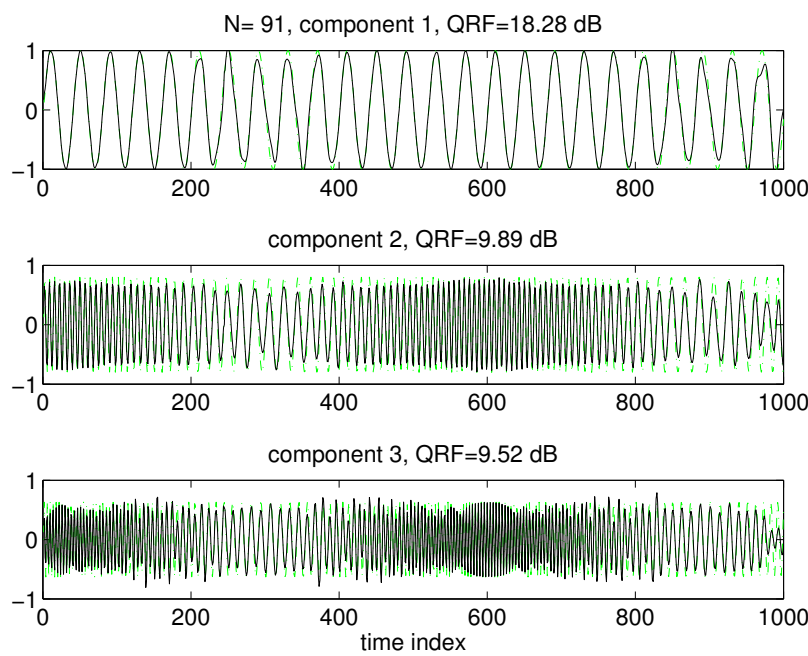

Fig. 7. Example 1: Sliding SSA decomposition of $s_{n}, r=3, L=40$ : the green plot is the reference and the black one is the reconstruction.

The QRF, computed through Eq. (7), is displayed in Fig. 7 which shows that the FM components can then be retrieved using the sliding SSA.

\section{Example 2}

The local character of the sliding SSA makes it adaptive to non-stationary components having different time supports. Consider a signal made of two components, displayed in Fig. 8(b) with linear frequency modulation and Gaussian amplitude modulation. SSA, applied to this signal, gives the components shown in Fig. 8(b). SSA extracts the component $y^{(1)}$ associated with the maximal peak of the signal energy spectral density and leaves the remaining of the signal energy to the second component $y^{(2)}$. SSA fails to recover the desired components. The sliding SSA, however, gives the components shown in Fig. 8(a), for $L=40$ and $W=131$. It is able to recover the signal components and gives high QRF. Numerical experiments showed that the sliding SSA decomposition is sensitive to the window length $W$. Nevertheless, acceptable results having high positive QRFs, can easily be obtained with a large set of $W$ values for which tuning is not a tedious task. 

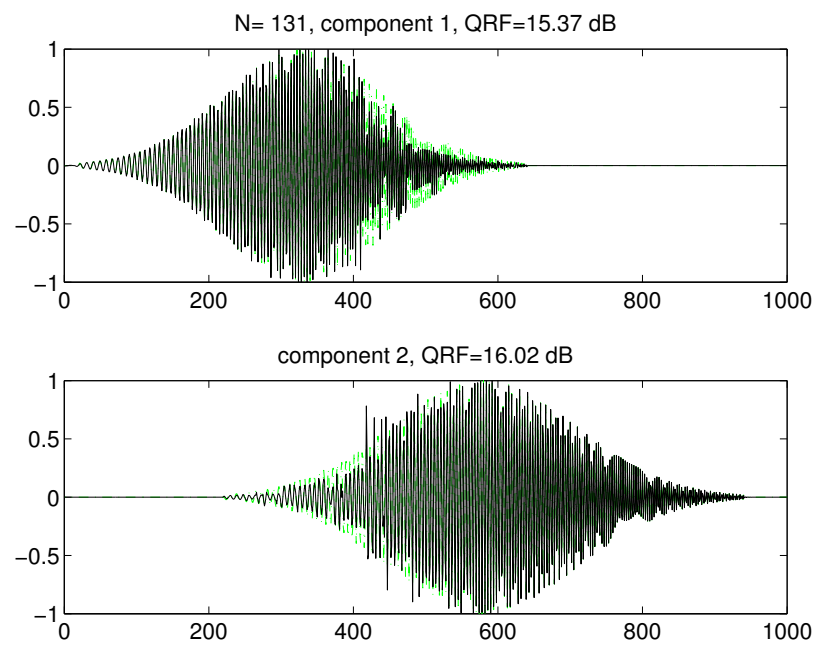

(a) sliding SSA
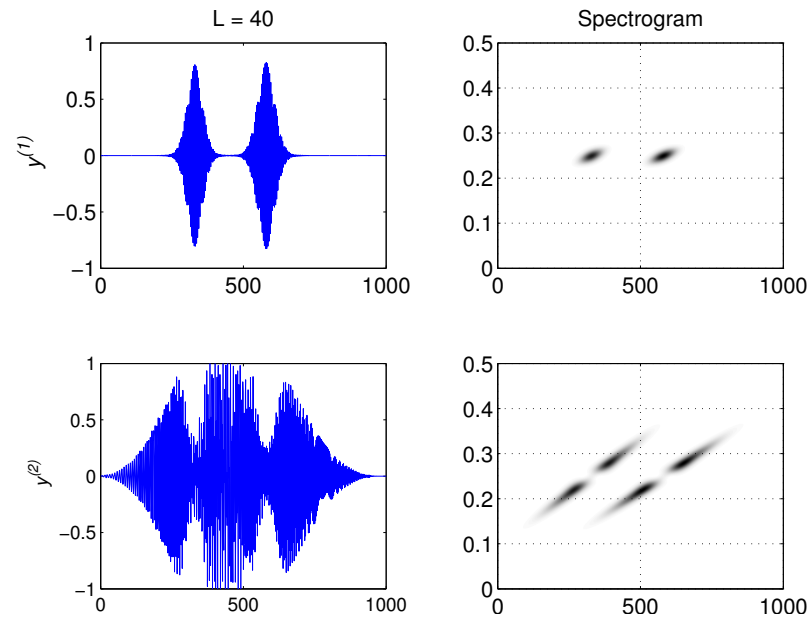

(b) automatic SSA

Fig. 8. Example 2: Sliding SSA decomposition and automatic SSA decomposition (b) of $s_{n}$ results $(r=2, L=40)$. In (a), the green line corresponds to the reference signal and the black line is its reconstruction.

\section{COMPARATIVE NUMERICAL RESUlS}

\section{A. SSA, Empirical Mode Decomposition and Sliding SSA}

As SSA, EMD [15] is a fully adaptive and data-driven method for analysis of multicomponent signals that assumes no a priori information about the linearity or stationarity of the signal. EMD decomposes the signal into a set of components called intrinsic mode functions (IMFs) such that their sum is equal to the original signal. The separation of two pure sinusoids from EMD perspective has been extensively studied in [38]. For this case, EMD and SSA behave as follows:

- When the amplitude of the high frequency component is higher than that of the low frequency, EMD and SSA act as linear filters with constant cutoff frequency that depend only on the number of sifting iterations for EMD, and the embedding dimension for SSA.

- When the amplitude of the high frequency component is smaller than that of the low frequency, EMD acts as a non-linear filter with a cutoff that depends on the amplitude ratio between the components. SSA however shows a symmetrical behavior regarding the amplitude ratio of the two components. SSA keeps the same constant value of the cutoff frequency.

Indeed, EMD is expected to extract progressively the different harmonics, starting with the highest frequency component and ending up with the lowest one. When the amplitude of the low frequency component gets higher than that of the high frequency one, the EMD separation fails because the first IMF is more likely to match the low frequency component.

In the particular case of a sum of a pure sinusoid and a chirp, it has been shown that SSA can separate between the two components when the amplitude of the pure sinusoid is higher than that of the chirp. SSA shows the same behavior as to whether the sinusoid frequency is higher or smaller than that of the chirp. Let's reconsider the following signal model

$$
\begin{aligned}
s_{n} & =\cos \left(2 \pi \lambda_{0} n\right)+H \cos (\varphi(n)) \\
\text { with } \varphi(n) & =2 \pi\left(\lambda_{1} n+(\delta \lambda / 2 N) n^{2}\right), n=1, \ldots, N
\end{aligned}
$$

Suppose $\lambda_{0}<\lambda_{1}+\delta \lambda$; SSA outperforms EMD when $H<1$ because as $H$ gets smaller than one, the first IMF will match the pure sinusoid, which is the low frequency component, and EMD will lead to a poor separation or a separation failure. The SSA separation succeeds everywhere for $H<1$, as shown in Fig. 3(b). EMD, However, is expected to outperform SSA when $H$ gets higher than one. In this respect, EMD and SSA have complementary separation capabilities.

In the case of general non-stationarity, Sliding SSA can provide SSA separation of general (AM-FM) component. Furthermore, EMD and Sliding SSA appear to have complementary separation capabilities of non-stationary components. Considering the signal model defined in Eq. (20) and the specified values of frequencies, it follows from the above discussion that on one hand the configuration of amplitudes which is more favorable to a Sliding SSA decomposition than an EMD decomposition is $a_{1}>a_{2}>a_{3}$, and on the other hand, the one which is more favorable for an EMD decomposition is $a_{1}<a_{2}<a_{3}$. However, while Sliding SSA algorithm is also meant to deal with signals of components having different time supports, as illustrated in section IV-C, EMD can only handle components having identical time supports.

\section{B. Sliding SSA and synchrosqueezing}

Synchrosqueezing [39] is a sharpening method designed to improve the energy localization of a Time Frequency Representation (TFR). Contrarily to the reassignment method [40], [41] which provides a non-invertible TFR, the synchrosqueezed transform allows signal reconstruction or mode extraction. To be used as an EMD-like tool, the synchrosqueezed transform has to be combined with a ridge detection algorithm, as in [42], [43]. Hence, the following experiment compares the mode extraction results obtained respectively by the proposed sliding SSA algorithm with the a mode extraction method based on the synchrosqueezed STFT.

1) Mode extraction using the synchrosqueezed STFT: Let $F_{x}^{h}(t, \omega)=\int_{\mathbb{R}} x(u) h(t-u)^{*} \mathbf{e}^{-j \omega u} d u$, be the STFT of a signal 
$x$ using analysis window $h$. Thus, the synchrosqueezed STFT of $x$ can be defined as [44]

$$
S_{x}(t, \omega)=\int_{\mathbb{R}} F_{x}^{h}(t, \Omega) \mathbf{e}^{j \Omega t} \delta(\omega-\hat{\omega}(t, \Omega)) d \Omega,
$$

where $\hat{\omega}(t, \omega)$ is a local instantaneous frequency estimator which can be provided by the frequency reassignment operator defined as [41], [45]

$$
\hat{\omega}(t, \omega)=\omega+\operatorname{Im}\left(\frac{F_{x}^{h^{\prime}}(t, \omega)}{F_{x}^{h}(t, \omega)}\right), \quad \text { with } h^{\prime}(t)=\frac{d h}{d t}(t) .
$$

Thus, for a given ridge curve denoted $\Omega_{i}(t)$, the i-th associated elementary component of the signal $x$ can be recovered as

$$
\hat{s}_{i}(t)=\frac{1}{h(0)} \int_{\Omega_{i}(t)-\Delta_{\omega} / 2}^{\Omega_{i}(t)+\Delta_{\omega} / 2} S_{x}(t, \omega) \frac{d \omega}{2 \pi}, \quad \text { if } h(0) \neq 0,
$$

while $\Delta_{\omega}$ sets the integration area around the ridge. In practice, $\Omega_{i}(t)$ can be obtained thanks to a ridge detector as proposed by Brevdo et al. in [43].

This technique finds the best frequency curve $\Omega(t)$ in the TFR, which maximizes the energy with a smooth constraint through a total variation term penalization obtained by

$$
\hat{\Omega}=\underset{\Omega}{\operatorname{argmax}} \int_{\mathbb{R}}\left|S_{x}(t, \Omega(t))\right|^{2} d t-\lambda \int_{\mathbb{R}}\left|\frac{d \Omega}{d t}(t)\right|^{2} d t,
$$

where $\lambda$ controls the importance of the smoothness of $\Omega$. When the ridges of several components have to be estimated, this method can be iterated after subtracting the energy located at the previously estimated ridge.

2) Numerical results: Now, we consider the signal made of three linear chirps, merged with a Gaussian white noise with a SNR equal to $25 \mathrm{~dB}$, as illustrated in Fig 9. We respectively apply the synchrosqueezing mode extraction method described in Section V-B1 and the proposed sliding SSA algorithm. Results presented in Fig. 10, shows that sliding SSA can obtain for each extracted component, better QRF values than those obtained with the synchrosqueezing mode extraction method. For this experiment, the settings of each method were empirically tuned, in order to provide the best results. For the sliding SSA method, we used a sliding analysis window of length equal to $W=101$ samples, the vector of the instantaneous number of components $r_{n}$ was manually defined such as $\max (r)=3$ and $L=39$. The reconstruction errors obtained by the synchrosqueezing method can be explained by ridge detection errors due to the presence of noise (despite several attempts for $\lambda$ ). This problem is clearly visible on the third reconstructed component ( $c f$. Fig. 10).

\section{Application on a real-world signal}

Now, we consider an audio signal of a cello musical instrument recorded at a sampling frequency $F_{s}=11050 \mathrm{~Hz}$, playing the note $G$ with a slight amplitude and frequency modulation. Hence, we propose to compare the results provided respectively by each of the studied methods (automatic SSA, sliding SSA, EMD and synchrosqueezed STFT) for the extraction of the four most prominent components (or partials). These components are located between $500 \mathrm{~Hz}$ and $3500 \mathrm{~Hz}$, as shown by Fig. 11(a). Sliding SSA achieves to correctly

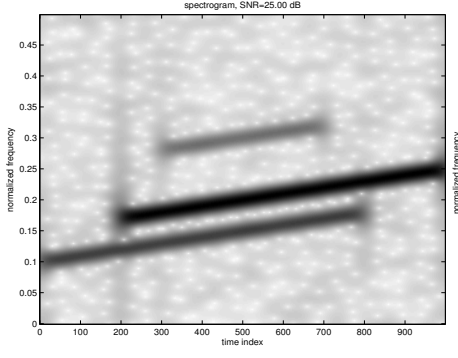

(a) spectrogram

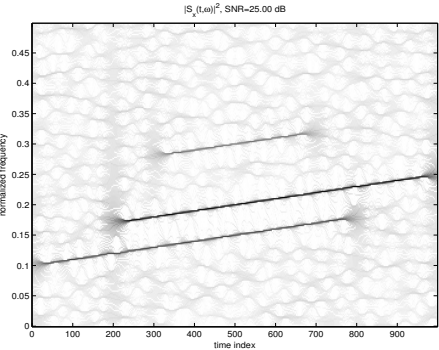

(b) $\left|S_{x}(t, \omega)\right|^{2}$
Fig. 9. TFRs of a multicomponent signal merged with a white Gaussian Noise $(\mathrm{SNR}=25 \mathrm{~dB})$.
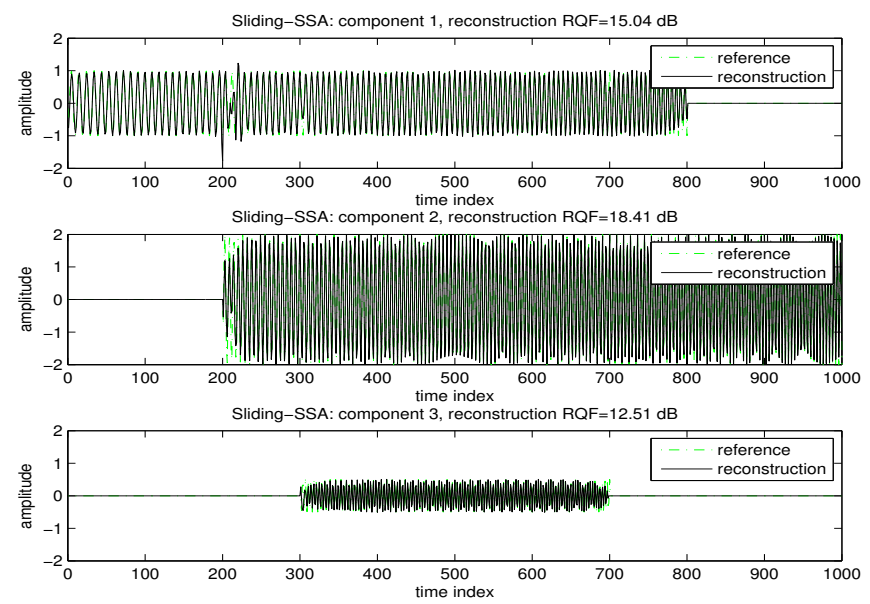

(a) sliding SSA
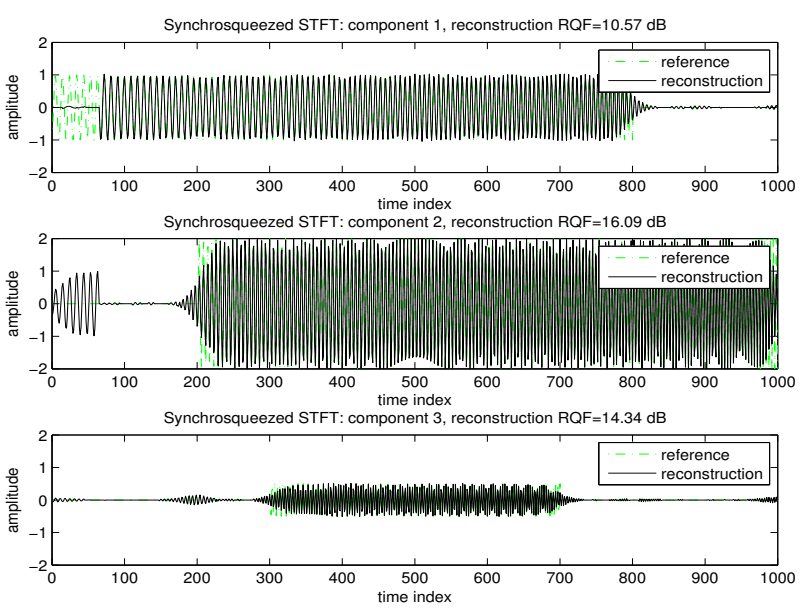

(b) synchrosqueezed STFT mode extraction [43]

Fig. 10. Mode extraction comparison between the sliding SSA and the synchrosqueezed STFT combined with [43] (SNR=25 dB).

extract the partials as illustrated by the resulting waveforms 11(b) and their corresponding spectrograms 11(c). To obtain this result, we used a sliding window of length $W=300$ with $L=150$. This result can informally be compared to Fig. 11(d) provided by the automatic SSA method and Fig. 11(e) provided by EMD which both fail to extract correctly all the partials of the analyzed signal. Hence, some components provided by SSA and EMD, contain energy located at 


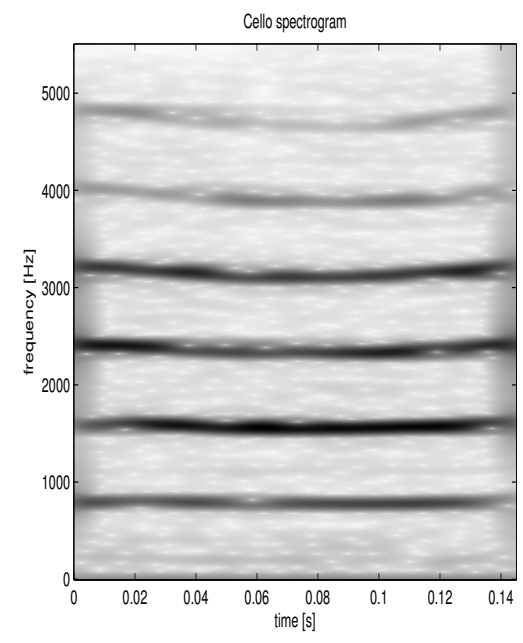

(a) reference spectrogram
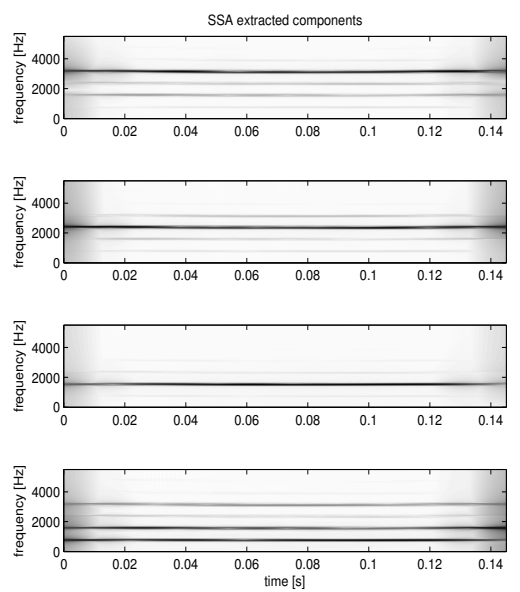

(d) automatic SSA (components spectrograms)
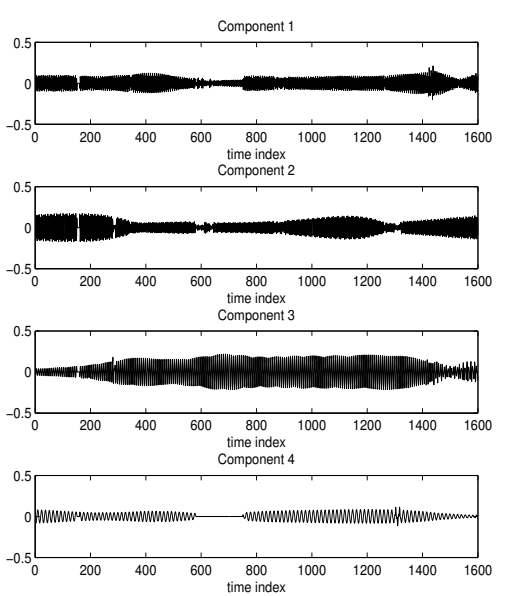

(b) sliding SSA (components waveforms)
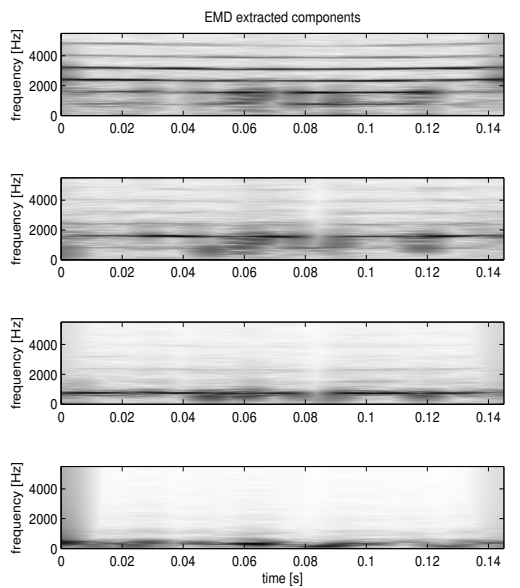

(e) EMD (components spectrograms)
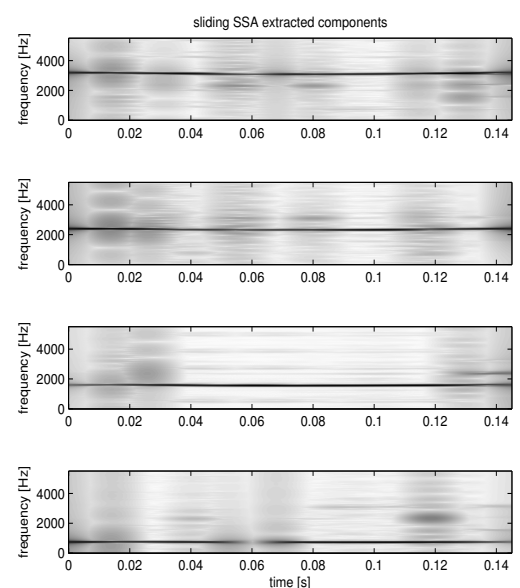

(c) sliding SSA (components spectrograms)
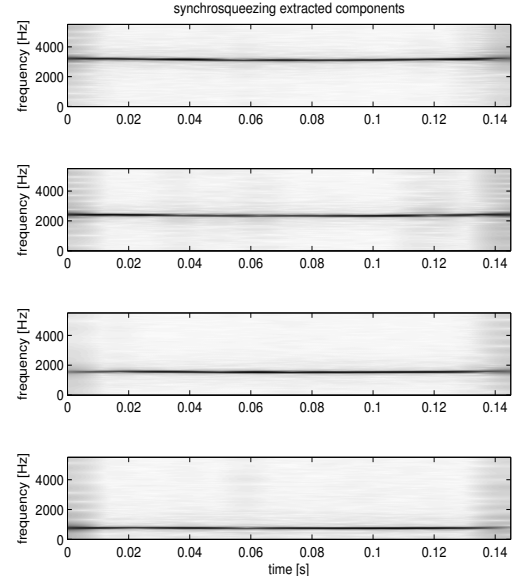

(f) synchrosqueezing (components spectrograms)

Fig. 11. Analysis of a signal produced by a cello playing the note G5 (fundamental frequency is about $784 \mathrm{~Hz}$ ). The components obtained with the new proposed sliding SSA algorithm (a)-(b) are compared with those provided by automatic SSA (d), EMD (e) and synchrosqueezing (f). In this experiment, each method is configured to provide $r=4$ components.

several frequencies due to the merging of several components. However, the synchrosqueezed STFT combined with [43] (cf. Fig. 11(f), provides the best component extraction results with less interferences than with the proposed sliding SSA method.

\section{CONCLUSION}

The SSA method was investigated from a theoretical and a practical point of view, with several enhancement proposals for automatic components grouping and non-stationary signals processing. A major contribution is the introduction of a new algorithm, called sliding SSA, which enhances the analysis of non-stationary signals thanks to a sliding analysis window as for the STFT. This new algorithm was compared with the classical SSA and with other state-of-the-art mode extraction methods: EMD and synchrosqueezing. Our numerical experiments show a clear advantage of the SSA over the EMD and many situation where the sliding SSA can obtain comparable results to the synchrosqueezing method. Future works consist in improving the robustness of the new proposed algorithm , to obtain a better tracking of the components. This could lead to the development more advanced applications for real-world signals.

\section{ACKNOWLEDGMENT}

This research was supported by the French ANR ASTRES project (ANR-13-BS03-0002-01).

\section{REFERENCES}

[1] D. Broomhead and G. King, "Extracting qualitative dynamics from experimental data," Physica D, vol. 20, pp. 217-236, 1986.

[2] R. Vautard and M. Ghil, "Singular Spectrum Analysis in nonlinear dynamics, with applications to paleoclimatic time series," Physica D, vol. 35, pp. 395-424, 1989.

[3] D. S. Broomhead and G. P. King, "On the qualitative analysis of experimental dynamical systems," in Nonlinear Phenomena and Chaos, S. Sarkar, Ed. Bristol, England: Adam Hilger, 1986, pp. 113-144.

[4] L. Yufeng and J. Saniie, "Singular spectrum analysis for trend extraction in ultrasonic backscattered echoes," in IEEE International Ultrasonics Symposium, Oct. 2015, pp. 1-4. 
[5] S. Enshaeifar, S. Kouchaki, C. Took, and S. Sanei, "Quaternion singular spectrum analysis of electroencephalogram with application in sleep analysis," IEEE Trans. Neural Syst. Rehabil. Eng., vol. 24, no. 1, pp. 57-67, Jan. 2016

[6] H. Hassani and Z. Ghodsi, "A glance at the applications of singular spectrum analysis in gene expression data," Biomolecular Detection and Quantification, vol. 4, pp. 17-21, 2015.

[7] F. Ghaderi, H. Mohseni, and S. Sanei, "Localizing heart sounds in respiratory signals using singular spectrum analysis," IEEE Trans. Biomed. Eng., vol. 58, no. 12, pp. 3360-3367, Dec. 2011.

[8] P. Flandrin, Time-Frequency/Time-Scale Analysis. Wavelet analysis and its applications. Academic Press, 1998, vol. 10.

[9] N. Golyandina and A. Shlemov, "Variations of Singular Spectrum Analysis for separability improvement: non-orthogonal decompositions of time series," Statistics and its interface, vol. 8, no. 3, pp. 277-294, 2014.

[10] N. Golyandina and A. Korobeynikov, "Basic Singular Spectrum Analysis and forecasting with R," Computational Statistics and Data Analysis, vol. 71, pp. 934-954, 2014.

[11] C. Rocco, "Singular Spectrum Analysis and forecasting of failure time series," Reliability Engineering and System Safety, vol. 114, pp. 126136, 2013.

[12] N. Golyandina, V. Nekrutkin, and A. Zhigljavsky, Analysis of Time Series Structure: SSA and Related Techniques. Chapman \& Hall/CRC, 2001.

[13] V. Nekrutkin, "Perturbation expansions of signal subspaces for long signals," J. Stat. Interface, vol. 3, pp. 297-319, 2010.

[14] H. Hassani, R. Mahmoudvand, and M. Zokaei, "Separability and window length in singular spectrum analysis," Comptes Rendus Mathematique, vol. 349, no. 17-18, pp. 987-990, 2011.

[15] N. E. Huang, Z. Shen, S. R. Long, M. C. Wu, H. H. Shih, Q. Zheng, N.C. Yen, C. C. Tung, and H. H. Liu, "The empirical mode decomposition and the Hilbert spectrum for nonlinear and non-stationary time series analysis," Proceedings of the Royal Society of London A: Mathematical, Physical and Engineering Sciences, vol. 454, no. 1971, pp. 903-995, 1998.

[16] I. Daubechies, J. Lu, and H.-T. Wu, "Synchrosqueezed wavelet transforms: An empirical mode decomposition-like tool," Applied and Computational Harmonic Analysis, vol. 30, no. 2, pp. 243-261, 2011.

[17] S. Serneels and T. Verdonck, "Principal component analysis for data containing outliers and missing elements," Computational Statistics \& Data Analysis, vol. 52, no. 3, pp. 1712-1727, 2008.

[18] G. Lin, N. Tang, and H. Wang, "Locally principal component analysis based on 11-norm maximisation," IET Image Processing, vol. 9, no. 2, pp. 91-96, 2015.

[19] N. Golyandina, "On the choice of parameters in singular spectrum analysis and related subspace-based methods," Statistics and Its Interface, vol. 3, pp. 259-279, 2010.

[20] G. W. Stewart, Introduction to Matrix Computations. Academic Press, 1973.

[21] D. G. Manolakis, V. K. Ingle, and S. M. Kogon, Statistical and Adaptive Signal Processing: Spectral Estimation, Signal Modeling, Adaptive Filtering and Array Processing. Artech House, 2005.

[22] H. Hassani, "Singular spectrum analysis: methodology and comparison," Journal of Data Science, vol. 5, no. 2, pp. 239-257, 2007.

[23] A. Alvarez-Meza, C. Acosta-Medina, and G. Castellanos-Dominguez, "Automatic Singular Spectrum Analysis for time-series decomposition," Proceedings of the European Symposium on Artificial Neural Networks, Computational Intelligence and Machine Learning, pp. 131-136, 2013.

[24] T. Alexandrov and N. E. Golyandina, "Automatic extraction and forecast of time series cyclic components within the framework of SSA," Proceedings of the 5th St.Petersburg Workshop on Simulation, 2005.

[25] T. Alexandrov, "A method of trend extraction using singular spectrum analysis," Statistical Journal, vol. 7, pp. 1-22, 2009.

[26] S. Sanei, M. Ghodsi, and H. Hassani, "An adaptive singular spectrum analysis approach to murmur detection from heart sounds," Medical engineering \& physics, vol. 33, no. 3, pp. 362-367, 2011.

[27] S. Sanei, T. K. Lee, and V. Abolghasemi, "A new adaptive line enhancer based on singular spectrum analysis," IEEE Trans. Biomed. Eng., vol. 59, no. 2, pp. 428-434, 2012.

[28] S. Kouchaki, S. Sanei, E. L. Arbon, and D.-J. Dijk, "Tensor based singular spectrum analysis for automatic scoring of sleep eeg," IEEE Trans. Neural Syst. Rehabil. Eng., vol. 23, no. 1, pp. 1-9, 2015.

[29] J. H. Ward, "Hierarchical grouping to optimize an objective function," $J A S A$, vol. 58, pp. 236-244, 1963.
[30] J. Harmouche, D. Fourer, F. Auger, P. Flandrin, and P. Borgnat, "One or two components ? the singular spectrum analysis answers," in Structured Low-Rank Approximation (SLRA'2015), Grenoble, France, Jun. 2015.

[31] F. Alonso and D. Salgado, "Analysis of the structure of vibration signals for tool wear detection," Mechanical Systems and Signal Processing, vol. 22, no. 3, pp. 735-748, 2008.

[32] M. Bilancia and F. Campobasso, "Airborne particulate matter and adverse health events: robust estimation of timescale effects," Classification as a Tool for Research, pp. 481-489, 2010.

[33] N. Golyandina and A. Zhigljavsky, Singular Spectrum Analysis for time series. Springer Science \& Business Media, 2013.

[34] G. A. F. Seber, Multivariate Observations. Hoboken, NJ: John Wiley \& Sons, Inc.

[35] D. Fourer, J. Harmouche, J. Schmitt, T. Oberlin, S. Meignen, F. Auger, and P. Flandrin, "The ASTRES toolbox for mode extraction of nonstationary multicomponent signals," in Proc. EUSIPCO, Kos island, Greece, Aug. 2017.

[36] E. Bozzo, R. Carniel, and D. Fasino, "Relationship between Singular Spectrum Analysis and Fourier analysis: Theory and application to the monitoring of volcanic activity," Computers and Mathematics with Applications, vol. 60, pp. 812-820, 2010.

[37] V. Georgescu, "A novel and effective approach to shape analysis: Nonparametric representation, de-noising and change-point detection, based on singular-spectrum analysis," Modeling Decision for Artificial Intelligence, vol. 6820, pp. 162-173, 2011.

[38] G. Rilling and P. Flandrin, "One or two frequencies? The Empirical Mode Decomposition answers," IEEE Trans. Signal Process, vol. 56, pp. 85-95, 2008.

[39] F. Auger, P. Flandrin, Y.-T. Lin, S. McLaughlin, S. Meignen, T. Oberlin, and $\mathrm{H}$. tieng $\mathrm{Wu}$, "Time-frequency reassignment and synchrosqueezing: An overview," Signal Processing Magazine, vol. 30, no. 6, pp. 32-41, Nov. 2013.

[40] K. Kodera, C. de Villedary, and R. Gendrin, "A new method for the numerical analysis of non-stationary signals," Physics of the Earth and Planetary Interiors, vol. 12, pp. 142-150, 1976

[41] F. Auger and P. Flandrin, "Improving the readability of time-frequency and time-scale representations by the reassignment method," IEEE Trans. Signal Process., vol. 43, no. 5, pp. 1068-1089, May 1995.

[42] S. Meignen, T. Oberlin, and S. McLaughlin, "A new algorithm for multicomponent signals analysis based on synchrosqueezing: with an application to signal sampling and denoising," IEEE Trans. Signal Process., vol. 60, no. 11, pp. 5787-5798, Nov. 2012.

[43] E. Brevdo, N. S. Fuckar, G. Thakur, and H.-T. Wu, "The synchrosqueezing algorithm: a robust analysis tool for signals with time-varying spectrum," arXiv id: 1105.0010, vol. 142, p. submitted, 2011.

[44] T. Oberlin, S. Meignen, and V. Perrier, "The Fourier-based synchrosqueezing transform," in Proc. IEEE ICASSP, 2014, pp. 315-319.

[45] D. Fourer, F. Auger, K. Czarnecki, S. Meignen, and P. Flandrin, "Chirp rate and instantaneous frequency estimation: application to recursive vertical synchrosqueezing," IEEE Signal Process. Lett., Jun. 2017. 


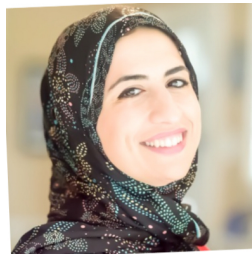

Jinane Harmouche completed her Ph.D in Physics at the University of Paris XI (Dec. 2014). She is now a postdoctoral researcher at the University of Waterloo in the department of Civil and Environmental Engineering and she is working as a part of the Stuctural Dynamics, Identification and Control group leaded by Professor Sriram Narasimhan. Her research work focuses on developing advanced datadriven approaches and signal processing techniques for the condition monitoring, fault detection and diagnostics of electro-mechanical systems.

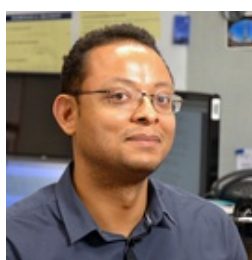

Dominique Fourer (M'11) received the M.Sc degree in Computer Sciences in 2008 and the M.Sc degree in Applied Mathematics in 2009, both from University of Bordeaux 1, France. He defended a $\mathrm{Ph} . \mathrm{D}$ thesis in 2013 in the fied of audio signal processing under the supervision of Prof. Sylvain Marchand at the Computer Sciences Research Laboratory of Bordeaux (LaBRI). He is now postdoctoral fellow (Chargé de Recherche) in the sound analysissynthesis team at the Institute of Research and Coordination Acoustic-Music (IRCAM) at Paris. His research interests focus in time-frequency analysis theory and applications in digital speech and audio signal processing related fields. As jazz musician (piano), he frequently plays with professional musicians in several music bands in France.

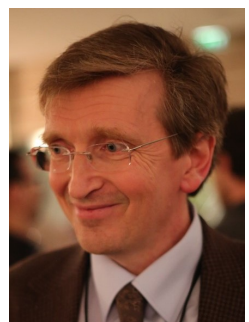

François Auger (M'93-SM'12) was born in SaintGermain-en-Laye, France, in 1966. He received the Engineer degree and the D.E.A. in signal processing and automatic control from the Ecole Nationale Supérieure de Mécanique de Nantes in 1988, and the Ph.D from the Ecole Centrale de Nantes in 1991. From 1993 to 2012, he was "Maître de Conférences" (assistant professor) at the IUT de Saint-Nazaire (Université de Nantes). He is now full professor at the same place. His current research interests include automatic control and diagnosis of electrical power systems, spectral analysis and time-frequency representation of non stationary signals. Dr. Auger is a member of the GdR720 CNRS "Information, Signal, Images et ViSion".

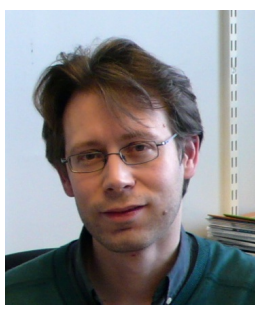

Pierre Borgnat (M'06) is a CNRS Senior Scientist, at the Laboratory of Physics, ENS de Lyon. Born in France in 1974, he was received at the École Normale Supérieure de Lyon in 1994 and as a Professeur Agrégé in Physical Sciences in 1997. He received the Ph.D. degree in Physics and Signal Processing in 2002. He worked in 20032004 at the ISR, IST (Lisbon, Portugal). He is a CNRS Chargé de Recherche since 2004, Directeur de Recherche since 2016. He is director of IXXI (Complex System Institute of Rhône-Alpes) since 2014. His research interests are in statistical signal processing, mainly graph signal processing, complex networks, nonstationary signals or scaling phenomena. He works on several applications of these signal processing methods: Internet traffic modeling and measurements, fluid mechanics, analysis of social data, transportation studies.

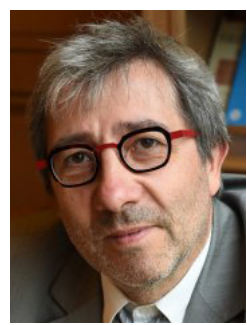

Patrick Flandrin (M'85-SM'01-F'02) received the engineer degree from ICPI Lyon, France, in 1978, and the Doct.-Ing. and Docteur dÉtat degrees from INP Grenoble, France, in 1982 and 1987, respectively. He joined CNRS in 1982, where he is currently Research Director. Since 1991, he has been with the Signals, Systems and Physics Group, within the Physics Department at ENS de Lyon, France. $\mathrm{He}$ is currently President of GRETSI, the French Association for Signal and Image Processing. His research interests include mainly nonstationary signal processing (with emphasis on time-frequency and time-scale methods), scaling stochastic processes and complex systems. He published over 250 research papers and authored one monograph in those areas. Dr. Flandrin was awarded the Philip Morris Scientific Prize in Mathematics (1991), the SPIE Wavelet Pioneer Award (2001), the Prix Michel Monpetit from the French Academy of sciences (2001) and the Silver Medal from CNRS (2010). Past Distinguished Lecturer of the IEEE Signal Processing Society (2010-2011), he is a Fellow of the IEEE (2002) and of EURASIP (2009), and he has been elected member of the French Academy of sciences in 2010. 